\title{
Ventilation in Relation to Arterial and Venous Blood Chemistry in Heart Disease
}

\author{
NIKOS GAZETOPOULOS, HYWEL DAVIES, AND DENNIS DEUCHAR
}

From the Cardiac Department, Guy's Hospital, London S.E.1

In a previous report (Gazetopoulos et al., 1966) of this study, the changes in hæmodynamics and ventilation with exercise were studied. In this paper we examine the parallel alterations in blood chemistry which take place in patients with various types of heart disease.

There are relatively few published reports of studies of the blood gases in relation to hyperventilation and dyspnoea in cardiac patients, and these are mainly concerned with mitral valve disease. Impairment of lung function, a potential cause of disturbed blood gases, has been shown to occur in such patients by Frank et al. (1953) and Riley et al. (1956) amongst others, and has been discussed by many authors including Rossier, Bühlmann, and Wiesinger (1960) and Arnott (1963).

In spite of this, Cullen et al. (1931) did not find any marked changes of $\mathrm{pH}$ and $\mathrm{PCO}_{2}$ in the arterial blood of exercising patients and concluded that the small changes observed were the result rather than the cause of hyperventilation. The same conclusion was reached by West et al. (1953) and Mauck and Shapiro (1961), though the former investigators noted abnormal blood gases in a minority of their patients. Mauck, Shapiro, and Patterson (1964), however, showed an increase of arterial $\mathrm{PCO}_{2}$ with exercise in 4 out of their 5 patients, sometimes significant in degree, and mentioned the possibility that this could be an additional stimulus to the respiratory centre. Arterial desaturation has also been observed in patients with mitral stenosis by Blount, McCord, and Anderson (1952) and Donald, Bishop, and Wade (1954), and the rise of blood lactic acid during exercise is said to be greater in patients with heart disease than in normal people (Huckabee and Judson, 1958; Donald et al., 1961).

More recently attention has turned to the venous side of the circulation (Armstrong et al., 1961; Riley, 1963). Although experiments in animals

Received March 12, 1965.
(Cropp and Comroe, 1961; Kao et al., 1963) make it unlikely that the venous blood chemistry acts as a normal controlling mechanism of ventilation, to our knowledge no observations have been published of direct measurements of venous chemistry in human subjects, and the possibility remains that such stimuli may exist, particularly in association with low output states.

The evaluation of humoral factors is of interest in mitral valve disease and left ventricular failure, in that they could serve as stimuli to ventilation additional to that provided by pulmonary congestion; their role in the determination of hyperventilation in patients without pulmonary venous hypertension, e.g. in pulmonary stenosis, is potentially more important. This study has been undertaken to obtain further information about the arterial and venous blood chemistry during exercise in various types of heart disease, in the hope that this might clarify the part played by chemical factors in producing hyperventilation.

\section{SUBJECTS AND METhodS}

These studies were performed during mild supine exercise at cardiac catheterization, and also during more severe exercise on a bicycle ergometer up to levels where dyspnœa was present. The clinical material consisted of 40 subjects, 5 of whom were normal; the remainder have been divided into three groups. Group I consists of 9 patients with left ventricular disease, group II of 14 patients in whom mitral stenosis was the dominant or sole lesion, and group III of 12 patients with isolated pulmonary stenosis. Details are given in Table I.

All were exercised during cardiac catheterization apart from Cases 26 and 40 in whom only resting catheterization data are available, and Cases 27 and 28 where no catheterization was performed and the clinical diagnosis of mild mitral stenosis was confirmed at operation. In these 4 patients studies were performed during 16 
TABLE I

CLINICAL DETAILS OF SUBJECTS STUDIED

\begin{tabular}{|c|c|c|c|c|c|c|c|}
\hline \multirow{2}{*}{$\begin{array}{c}\text { Group } \\
\text { Normal }\end{array}$} & \multicolumn{3}{|c|}{$\begin{array}{c}\text { Case No. sex, and age } \\
\text { (yr.) }\end{array}$} & \multirow{2}{*}{$\begin{array}{l}\begin{array}{c}\text { BSA } \\
\left(\mathrm{m.}{ }^{2}\right)\end{array} \\
1.45 \\
1.60 \\
1.70 \\
1.20 \\
1.50\end{array}$} & \multirow{2}{*}{$\begin{array}{c}\begin{array}{c}\text { Oxygen } \\
\text { capacity } \\
\text { (vol. \%) }\end{array} \\
18 \cdot 2 \\
15 \cdot 5 \\
20 \cdot 9 \\
15 \cdot 5 \\
18 \cdot 2 \\
\end{array}$} & \multirow{2}{*}{$\begin{array}{c}\text { Disability } \\
0 \\
0 \\
0 \\
0 \\
0\end{array}$} & \multirow{2}{*}{$\begin{array}{l}\text { Diagnosis } \\
\text { Normal } \\
\text { Normal } \\
\text { Normal } \\
\text { Normal } \\
\text { Normal }\end{array}$} \\
\hline & $\begin{array}{l}1 \\
2 \\
3 \\
4 \\
5 \\
\end{array}$ & $\begin{array}{l}\mathbf{F} \\
\mathbf{F} \\
\mathbf{M} \\
\mathbf{M} \\
\mathbf{M}\end{array}$ & $\begin{array}{l}18 \\
17 \\
29 \\
14 \\
11 \\
\end{array}$ & & & & \\
\hline $\begin{array}{c}\text { I: Left } \\
\text { ventricular } \\
\text { disease }\end{array}$ & $\begin{array}{r}6 \\
7 \\
8 \\
9 \\
10 \\
11 \\
12 \\
13 \\
14\end{array}$ & $\begin{array}{l}\mathbf{M} \\
\mathbf{M} \\
\mathbf{M} \\
\mathbf{F} \\
\mathbf{M} \\
\mathbf{M} \\
\mathbf{F} \\
\mathbf{M}\end{array}$ & $\begin{array}{l}26 \\
24 \\
62 \\
59 \\
50 \\
61 \\
33 \\
35 \\
63\end{array}$ & $\begin{array}{l}1.80 \\
1.60 \\
1.90 \\
1.75 \\
1.60 \\
1.70 \\
1.75 \\
1.50 \\
1.67\end{array}$ & $\begin{array}{l}16 \cdot 1 \\
17 \cdot 9 \\
18 \cdot 2 \\
16 \cdot 7 \\
16 \cdot 9 \\
22 \cdot 8 \\
22 \cdot 0 \\
18 \cdot 0 \\
16 \cdot 3\end{array}$ & $\begin{array}{l}2 \\
3-\mathrm{A} \\
2 \\
3-\mathrm{A} \\
3-\mathrm{B} \\
3-\mathrm{A} \\
2 \\
3-\mathrm{A} \\
4\end{array}$ & $\begin{array}{l}\text { AS } \\
\text { AS } \\
\text { AS } \\
\text { AS } \\
\text { AS } \\
\text { AS } \\
\text { AS (complete heart block) } \\
\text { Cardiomyopathy } \\
\text { Left ventricular failure, emphysema (AF) }\end{array}$ \\
\hline $\begin{array}{l}\text { II: Mitral } \\
\text { stenosis }\end{array}$ & $\begin{array}{l}15 \\
16 \\
17 \\
18 \\
19 \\
20 \\
21 \\
22 \\
23 \\
24 \\
25 \\
26 \\
27 \\
28\end{array}$ & $\begin{array}{l}\mathbf{F} \\
\mathbf{M} \\
\mathbf{F} \\
\mathbf{F} \\
\mathbf{F} \\
\mathbf{M} \\
\mathbf{M} \\
\mathbf{F} \\
\mathbf{F} \\
\mathbf{M} \\
\mathbf{F} \\
\mathbf{M} \\
\mathbf{F}\end{array}$ & $\begin{array}{l}47 \\
39 \\
33 \\
51 \\
40 \\
46 \\
26 \\
35 \\
46 \\
43 \\
43 \\
22 \\
49 \\
40\end{array}$ & $\begin{array}{l}1.35 \\
2.00 \\
1.50 \\
1.55 \\
1.50 \\
1.70 \\
2.00 \\
1.50 \\
1.50 \\
1.65 \\
1.70 \\
1.75 \\
1.85 \\
1.50\end{array}$ & $\begin{array}{l}17 \cdot 8 \\
18 \cdot 4 \\
14 \cdot 6 \\
17 \cdot 6 \\
17 \cdot 6 \\
19 \cdot 2 \\
22 \cdot 3 \\
10 \cdot 2 \\
20 \cdot 9 \\
16 \cdot 9 \\
22 \cdot 1 \\
16 \cdot 3 \\
14 \cdot 7 \\
15 \cdot 6\end{array}$ & $\begin{array}{l}3-\mathbf{B} \\
3-\mathbf{A} \\
3-\mathbf{A} \\
3-\mathbf{B} \\
3-\mathbf{B} \\
3-\mathbf{B} \\
3-\mathbf{A} \\
3-\mathbf{B} \\
3-\mathbf{B} \\
2 \\
3-\mathbf{A} \\
2 \\
3-\mathbf{A} \\
3-\mathbf{A}\end{array}$ & $\begin{array}{l}\text { MS } \\
\text { MS (AF) } \\
\text { MS (AF) } \\
\text { MS, AI (AF) } \\
\text { MS, MI, TI (AF) } \\
\text { MS (AF), TI (AF) } \\
\text { MS, MI, TI APs } \\
\text { MS, MI, hypertension emphysema } \\
\text { MS } \\
\text { MS } \\
\text { MS (AF) } \\
\text { MS }\end{array}$ \\
\hline $\begin{array}{c}\text { III: Pul- } \\
\text { monary } \\
\text { stenosis }\end{array}$ & $\begin{array}{l}29 \\
30 \\
31 \\
32 \\
33 \\
34 \\
35 \\
36 \\
37 \\
38 \\
39 \\
40\end{array}$ & $\begin{array}{l}\mathbf{F} \\
\mathbf{M} \\
\mathbf{F} \\
\mathbf{F} \\
\mathbf{F} \\
\mathbf{M} \\
\mathbf{M} \\
\mathbf{M} \\
\mathbf{M} \\
\mathbf{M} \\
\mathbf{M} \\
\mathbf{M}\end{array}$ & $\begin{array}{r}29 \\
7 \\
21 \\
44 \\
14 \\
19 \\
30 \\
15 \\
32 \\
36 \\
58 \\
13\end{array}$ & $\begin{array}{l}1.42 \\
1.00 \\
1.30 \\
1.70 \\
1.45 \\
1.85 \\
1.90 \\
1.95 \\
1.95 \\
1.75 \\
1.95 \\
1.25\end{array}$ & $\begin{array}{l}15.5 \\
14.4 \\
20.3 \\
18.8 \\
20.6 \\
19.8 \\
17.6 \\
21.3 \\
20.6 \\
20.9 \\
20.9 \\
18.7\end{array}$ & $\begin{array}{l}\text { 3-A } \\
\mathbf{I} \\
3-\mathbf{B} \\
3-\mathbf{A} \\
2 \\
\mathbf{I} \\
\mathbf{I} \\
\mathbf{I} \\
\mathbf{I} \\
2 \\
3-\mathbf{B} \\
3-\mathbf{A}\end{array}$ & $\begin{array}{l}\text { PS } \\
\text { PS } \\
\text { PS } \\
\text { PS } \\
\text { PS } \\
\text { PS } \\
\text { PS } \\
\text { PS } \\
\text { PS } \\
\text { PS } \\
\text { PS } \\
\text { PS }\end{array}$ \\
\hline
\end{tabular}

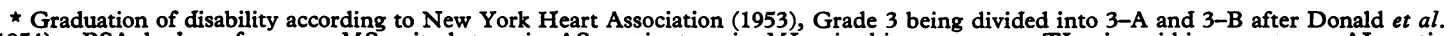
(1954). BSA, body surface area; MS, mitral stenosis; AS, aortic stenosis; MI, mitral incompetence; TI, tricuspid incompetence; AI, aortic incompetence; $\mathrm{AF}$, atrial fibrillation; $\mathrm{PS}$, pulmonary stenosis.

The techniques of cardiac catheterization and the hæmodynamic studies were similar to those described in a previous paper (Gazetopoulos et al., 1966), exercise having been carried out in the supine position by means of a spring-loaded leg exerciser. Arterial blood samples were withdrawn at rest, in the third to fourth minute, and the eighth to tenth minute of exercise. They were analysed for $\mathrm{pH}, \mathrm{PCO}_{2}$, bicarbonate, oxygen saturation, lactate, and pyruvate. Samples were also withdrawn simultaneously from the pulmonary artery and analysed for $\mathrm{pH}, \mathrm{PCO}_{2}$, bicarbonate, and oxygen saturation. The $\mathrm{pH}$ and $\mathrm{PCO}_{2}$ were measured by the micro-Astrup method, the oxygen saturations spectrophotometrically, and the lactate and pyruvate by enzymatic methods. Details are given elsewhere (Davies and Gazetopoulos, 1965).

On a separate occasion the 4 patients mentioned above and 6 others were exercised in the upright position on a bicycle ergometer, the initial level being $200 \mathrm{~kg}$./ min. Where possible the level was increased by 200 $\mathrm{kg}$./min. at intervals of 10 minutes, exercise being terminated when dyspnœea and fatigue dictated this.

C
These patients were thus stressed to their maximum working capacity. Ventilation, oxygen uptake, heart rate, and changes in arterial blood chemistry were measured as previously described (Davies, Gazetopoulos, and Oliver, 1965), blood samples being again withdrawn in the third to fourth minute and eighth to tenth minute of each level. If exercise had to be stopped, a sample was obtained just before this point. It was impossible to measure ventilation in all subjects at the critical levels, but their reactions to the procedures were assessed as carefully as possible.

An untoward reaction occurred in only one patient who developed evidence of pulmonary odema (Case 16). This responded well to the usual measures, and he has since had a successful mitral valvotomy.

\section{Results}

A. Mild Supine Exercise. Table II gives the ventilatory and hæmodynamic findings and Table III the arterial and mixed venous blood composition 
TABLE II

HAMODYNAMICS AND VENTILATION AT REST AND ON EXERCISE IN PATIENTS STUDIED

\begin{tabular}{|c|c|c|c|c|c|c|c|c|c|c|c|c|}
\hline \multirow[b]{2}{*}{ Group } & \multirow[b]{2}{*}{$\begin{array}{l}\text { Case } \\
\text { No. }\end{array}$} & \multirow{2}{*}{$\begin{array}{c}\dot{\mathrm{V}}_{2} \\
\text { (ml./min.) } \\
\text { STPD }\end{array}$} & \multirow[b]{2}{*}{$\begin{array}{c}\text { Heart } \\
\text { rate }\end{array}$} & \multirow{2}{*}{$\begin{array}{c}\begin{array}{c}\text { A-V } \\
\text { diff. } \\
\text { (vol. } \% \text { ) }\end{array} \\
\end{array}$} & \multirow[b]{2}{*}{$\begin{array}{c}\mathrm{CO} \\
(1 . / \mathrm{min} .) \\
\end{array}$} & \multicolumn{4}{|c|}{ Pressures (mm. $\mathbf{H g}$ ) } & \multirow{2}{*}{$\underset{\text { BTPS }}{\dot{\mathbf{v}}}$} & \multirow[b]{2}{*}{$\begin{array}{c}\text { Resp. } \\
\text { rate }\end{array}$} & \multirow[b]{2}{*}{ VE } \\
\hline & & & & & & $\begin{array}{l}\text { PCV } \\
\text { mean }\end{array}$ & $\begin{array}{c}\text { PA } \\
\text { s/d/mean }\end{array}$ & $\begin{array}{l}\text { RV } \\
\text { s/d }\end{array}$ & $\begin{array}{c}\text { Syst. } \\
\text { s/d/mean }\end{array}$ & & & \\
\hline \multirow[t]{5}{*}{ Normal } & $1 \underset{\mathbf{E}}{\mathbf{R}}$ & $\begin{array}{l}200 \\
500\end{array}$ & $\begin{array}{l}100 \\
125\end{array}$ & $\begin{array}{l}3 \cdot 3 \\
6 \cdot 7\end{array}$ & $\begin{array}{l}6 \cdot 1 \\
7 \cdot 5\end{array}$ & 7 & $\begin{array}{l}18 / 10 / 13 \\
20 / 10 / 15\end{array}$ & $23 / 5$ & $\begin{array}{l}115 / 75 / 85 \\
100 / 65 / 85\end{array}$ & $\begin{array}{r}5 \cdot 6 \\
15 \cdot 2\end{array}$ & $\begin{array}{l}17 \\
20\end{array}$ & $\begin{array}{l}2 \cdot 8 \\
3 \cdot 0\end{array}$ \\
\hline & $2 \underset{\mathrm{E}}{\mathrm{R}}$ & $\begin{array}{l}250 \\
595\end{array}$ & $\begin{array}{r}75 \\
115\end{array}$ & $\begin{array}{l}3.0 \\
7.0\end{array}$ & $\begin{array}{l}8 \cdot 3 \\
8 \cdot 5\end{array}$ & $\begin{array}{l}12 \\
12\end{array}$ & $\begin{array}{l}26 / 10 / 14 \\
26 / 10 / 14\end{array}$ & $\begin{array}{l}28 / 7 \\
25 / 5\end{array}$ & $\begin{array}{l}130 / 60 / 80 \\
170 / 90 / 115\end{array}$ & $\begin{array}{r}4 \cdot 6 \\
17 \cdot 2\end{array}$ & $\begin{array}{l}14 \\
20\end{array}$ & $\begin{array}{l}1.8 \\
2.9\end{array}$ \\
\hline & $3 \underset{\mathbf{E}}{\mathbf{R}}$ & $\begin{array}{l}180 \\
470\end{array}$ & $\begin{array}{r}72 \\
120\end{array}$ & $\begin{array}{l}2 \cdot 8 \\
6 \cdot 4\end{array}$ & $\begin{array}{l}7 \cdot 0 \\
7 \cdot 4\end{array}$ & $\begin{array}{l}10 \\
12\end{array}$ & $\begin{array}{l}25 / 10 / 20 \\
30 / 10 / 22\end{array}$ & $30 / 9$ & $\begin{array}{l}125 / 50 / 75 \\
110 / 65 / 80\end{array}$ & $\begin{array}{r}6 \cdot 4 \\
14.6\end{array}$ & $\begin{array}{l}13 \\
15\end{array}$ & $\begin{array}{l}3 \cdot 5 \\
3 \cdot 1\end{array}$ \\
\hline & ${ }_{\mathbf{E}}^{\mathbf{R}}$ & $\begin{array}{l}175 \\
280\end{array}$ & $\begin{array}{l}60 \\
94\end{array}$ & $\begin{array}{l}2 \cdot 9 \\
3 \cdot 2\end{array}$ & $\begin{array}{l}6 \cdot 0 \\
8 \cdot 7\end{array}$ & 10 & $\underline{25 / 8 / 14}$ & 30/8 & $\begin{array}{r}95 / 50 / 75 \\
110 / 60 / 75\end{array}$ & $\begin{array}{l}4 \cdot 1 \\
6 \cdot 6\end{array}$ & $\begin{array}{l}16 \\
20\end{array}$ & $\begin{array}{l}2 \cdot 3 \\
2 \cdot 3\end{array}$ \\
\hline & $\begin{array}{r}5 \mathbf{R} \\
\mathbf{E} \\
\end{array}$ & 170 & $\frac{76}{\square}$ & $\frac{3 \cdot 1}{-}$ & 5.5 & 6 & $\begin{array}{l}22 / 8 / 12 \\
30 / 15 / 18\end{array}$ & $25 / 2$ & $\begin{array}{l}115 / 70 / 85 \\
150 / 75 / 95 \\
\end{array}$ & $\frac{9 \cdot 0}{-}$ & 24 & $\stackrel{5 \cdot 2}{-}$ \\
\hline \multirow{9}{*}{$\begin{array}{l}\text { I: Left } \\
\text { ventricular } \\
\text { disease }\end{array}$} & $6 \underset{\mathbf{E}}{\mathbf{R}}$ & $\begin{array}{l}265 \\
720\end{array}$ & $\begin{array}{r}60 \\
100\end{array}$ & $\begin{array}{l}5 \cdot 0 \\
7 \cdot 4\end{array}$ & $\begin{array}{l}5 \cdot 3 \\
9 \cdot 7\end{array}$ & $\begin{array}{l}14 \\
18\end{array}$ & $\begin{array}{l}28 / 18 / 20 \\
28 / 18 / 20\end{array}$ & $\stackrel{28 / 8}{-}$ & $\begin{array}{l}100 / 60 / 80 \\
165 / 80 / 105\end{array}$ & $\begin{array}{r}5 \cdot 5 \\
15 \cdot 4\end{array}$ & $\begin{array}{l}10 \\
14\end{array}$ & $\begin{array}{l}2 \cdot 1 \\
2 \cdot 1\end{array}$ \\
\hline & $7 \underset{\mathbf{E}}{\mathbf{R}}$ & $\begin{array}{l}200 \\
405\end{array}$ & $\begin{array}{r}75 \\
120\end{array}$ & $\begin{array}{l}3.4 \\
4.8\end{array}$ & $\begin{array}{l}5 \cdot 9 \\
8 \cdot 4\end{array}$ & $\begin{array}{r}8 \\
10\end{array}$ & $\begin{array}{l}20 / 10 / 14 \\
25 / 10 / 18\end{array}$ & $\stackrel{25 / 5}{-}$ & $\begin{array}{r}95 / 65 / 75 \\
100 / 65 / 85\end{array}$ & $\begin{array}{r}6 \cdot 0 \\
11 \cdot 2\end{array}$ & $\begin{array}{l}18 \\
20\end{array}$ & $\begin{array}{l}3 \cdot 0 \\
2 \cdot 8\end{array}$ \\
\hline & $8 \underset{\mathrm{E}}{\mathrm{R}}$ & $\begin{array}{l}190 \\
270\end{array}$ & $\begin{array}{l}55 \\
83\end{array}$ & $\begin{array}{l}5 \cdot 2 \\
4 \cdot 1\end{array}$ & $\begin{array}{l}3.7 \\
5.9\end{array}$ & $\begin{array}{l}17 \\
19\end{array}$ & $\begin{array}{l}50 / 20 / 35 \\
55 / 20 / 35\end{array}$ & 50/5 & $\begin{array}{l}100 / 60 / 75 \\
110 / 55 / 80\end{array}$ & $\begin{array}{l}6 \cdot 1 \\
8 \cdot 2\end{array}$ & $\begin{array}{l}12 \\
14\end{array}$ & $\begin{array}{l}3 \cdot 2 \\
3 \cdot 0\end{array}$ \\
\hline & $9 \underset{\mathbf{E}}{\mathbf{R}}$ & $\begin{array}{l}245 \\
580\end{array}$ & $\begin{array}{l}63 \\
80\end{array}$ & $\begin{array}{l}4 \cdot 6 \\
7 \cdot 7\end{array}$ & $\begin{array}{l}5 \cdot 5 \\
7 \cdot 6\end{array}$ & $\begin{array}{l}12 \\
18\end{array}$ & $\begin{array}{l}28 / 13 / 18 \\
40 / 25 / 30\end{array}$ & $26 / 6$ & $\begin{array}{l}105 / 60 / 85 \\
130 / 75 / 100\end{array}$ & $\begin{array}{r}7 \cdot 2 \\
13 \cdot 9\end{array}$ & $\begin{array}{l}11 \\
14\end{array}$ & $\begin{array}{l}2.9 \\
2 \cdot 4\end{array}$ \\
\hline & $10 \underset{E}{R}$ & $\begin{array}{l}210 \\
300\end{array}$ & $\begin{array}{r}94 \\
102\end{array}$ & $\begin{array}{l}4 \cdot 4 \\
5 \cdot 4\end{array}$ & $\begin{array}{l}4 \cdot 7 \\
5 \cdot 5\end{array}$ & $\begin{array}{l}15 \\
18\end{array}$ & $\begin{array}{l}35 / 15 / 20 \\
36 / 12 / 22\end{array}$ & $35 / 6$ & $\begin{array}{l}140 / 80 / 100 \\
150 / 90 / 110\end{array}$ & $\begin{array}{l}5.5 \\
7.7\end{array}$ & $\begin{array}{l}12 \\
14\end{array}$ & $\begin{array}{l}2.7 \\
2.6\end{array}$ \\
\hline & $11 \underset{\mathbf{E}}{\mathbf{R}}$ & $\begin{array}{l}210 \\
330\end{array}$ & $\begin{array}{l}56 \\
84\end{array}$ & $\begin{array}{l}5 \cdot 7 \\
6 \cdot 7\end{array}$ & $\begin{array}{l}3.65 \\
5.0\end{array}$ & $\begin{array}{l}12 \\
28\end{array}$ & $\begin{array}{l}50 / 20 / 26 \\
80 / 35 / 42\end{array}$ & $\stackrel{55 / 8}{-}$ & $\begin{array}{l}150 / 70 / 100 \\
155 / 75 / 100\end{array}$ & $\begin{array}{r}6.2 \\
11.5\end{array}$ & $\begin{array}{r}81 \\
21\end{array}$ & $\begin{array}{l}2.9 \\
3.5\end{array}$ \\
\hline & $12 \underset{E}{R}$ & $\begin{array}{l}290 \\
665\end{array}$ & $\begin{array}{l}55 \\
60\end{array}$ & $\begin{array}{l}5 \cdot 7 \\
9 \cdot 4\end{array}$ & $\begin{array}{l}5 \cdot 1 \\
7 \cdot 1\end{array}$ & $\begin{array}{l}16 \\
14\end{array}$ & $\begin{array}{l}36 / 16 / 23 \\
57 / 19 / 33\end{array}$ & $36 / 15$ & $\begin{array}{l}145 / 60 / 88 \\
158 / 66 / 94\end{array}$ & $\begin{array}{r}6 \cdot 1 \\
16 \cdot 5\end{array}$ & $\begin{array}{l}13 \\
15\end{array}$ & $\begin{array}{l}2 \cdot 1 \\
2 \cdot 5\end{array}$ \\
\hline & $13 \underset{\mathbf{E}}{\mathbf{R}}$ & $\begin{array}{l}180 \\
270\end{array}$ & $\begin{array}{l}65 \\
75\end{array}$ & $\begin{array}{l}4 \cdot 1 \\
6 \cdot 2\end{array}$ & $\begin{array}{l}4 \cdot 4 \\
4 \cdot 4\end{array}$ & 10 & $\begin{array}{l}28 / 12 / 19 \\
35 / 18 / 24\end{array}$ & $28 / 8$ & $\begin{array}{l}100 / 50 / 70 \\
110 / 65 / 82\end{array}$ & $\begin{array}{l}5 \cdot 4 \\
9 \cdot 6\end{array}$ & $\begin{array}{l}13 \\
23\end{array}$ & $\begin{array}{l}3 \cdot 0 \\
3.5\end{array}$ \\
\hline & $14 \underset{E}{R}$ & $\begin{array}{l}224 \\
295 \\
\end{array}$ & $\begin{array}{l}80 \\
85\end{array}$ & $\begin{array}{l}4.9 \\
6.4\end{array}$ & $\begin{array}{l}4 \cdot 6 \\
4 \cdot 6\end{array}$ & 20 & $\begin{array}{l}55 / 25 / 35 \\
67 / 30 / 50\end{array}$ & $\stackrel{55 / 66}{-}$ & $140 / 60 / 80$ & $\begin{array}{r}7 \cdot 1 \\
11 \cdot 2\end{array}$ & $\begin{array}{l}26 \\
21\end{array}$ & $\begin{array}{l}3.2 \\
3.8\end{array}$ \\
\hline \multirow[t]{12}{*}{$\begin{array}{l}\text { II: Mitral } \\
\text { stenosis }\end{array}$} & $15 \underset{E}{\mathbf{R}}$ & $\begin{array}{l}170 \\
255\end{array}$ & $\begin{array}{l}106 \\
136\end{array}$ & $\begin{array}{l}3 \cdot 0 \\
4 \cdot 2\end{array}$ & $\begin{array}{l}5 \cdot 6 \\
6 \cdot 1\end{array}$ & $\begin{array}{l}21 \\
26\end{array}$ & $\begin{array}{l}41 / 23 / 29 \\
52 / 31 / 38\end{array}$ & 43/8 & $\begin{array}{l}120 / 72 / 95 \\
157 / 90 / 115\end{array}$ & $\begin{array}{l}5.5 \\
7.5\end{array}$ & $\begin{array}{l}20 \\
24\end{array}$ & $\begin{array}{l}3.2 \\
2.9\end{array}$ \\
\hline & $16 \underset{E}{R}$ & $\begin{array}{l}260 \\
520\end{array}$ & $\begin{array}{r}88 \\
150\end{array}$ & $\begin{array}{r}6 \cdot 0 \\
10 \cdot 4\end{array}$ & $\begin{array}{l}4 \cdot 4 \\
5 \cdot 0\end{array}$ & $\begin{array}{l}35 \\
50\end{array}$ & $\begin{array}{l}90 / 40 / 65 \\
130 / 70 / 80\end{array}$ & $\stackrel{90 / 13}{-}$ & $\begin{array}{l}125 / 80 / 95 \\
150 / 75 / 100\end{array}$ & $\begin{array}{l}11 \cdot 2 \\
32 \cdot 4\end{array}$ & $\begin{array}{l}14 \\
21\end{array}$ & $\begin{array}{l}4 \cdot 3 \\
6 \cdot 2\end{array}$ \\
\hline & $17 \underset{\mathrm{E}}{\mathrm{R}}$ & $\begin{array}{l}215 \\
410\end{array}$ & $\begin{array}{r}94 \\
108\end{array}$ & $\begin{array}{l}4 \cdot 7 \\
5 \cdot 2\end{array}$ & $\begin{array}{l}4.6 \\
7.9\end{array}$ & $\begin{array}{l}23 \\
30\end{array}$ & $\begin{array}{l}60 / 25 / 40 \\
75 / 40 / 50\end{array}$ & $\begin{array}{l}60 / 6 \\
75 / 8\end{array}$ & $\begin{array}{l}140 / 65 / 90 \\
150 / 70 / 100\end{array}$ & $\begin{array}{r}5 \cdot 3 \\
12 \cdot 3\end{array}$ & $\begin{array}{l}12 \\
28\end{array}$ & $\begin{array}{l}2.5 \\
3 \cdot 0\end{array}$ \\
\hline & $18 \underset{\mathbf{E}}{\mathbf{R}}$ & $\begin{array}{l}180 \\
450\end{array}$ & $\begin{array}{r}60 \\
104\end{array}$ & $\begin{array}{l}5 \cdot 8 \\
9 \cdot 1\end{array}$ & $\begin{array}{l}3 \cdot 1 \\
5 \cdot 0\end{array}$ & $\begin{array}{l}20 \\
28\end{array}$ & $\begin{array}{l}50 / 20 / 28 \\
75 / 35 / 45\end{array}$ & $\begin{array}{l}50 / 8 \\
75 / 10\end{array}$ & $\begin{array}{c}80 / 90 / 65 \\
140 / 75 / 100\end{array}$ & $\begin{array}{r}4 \cdot 9 \\
13 \cdot 3\end{array}$ & $\begin{array}{l}15 \\
24\end{array}$ & $\begin{array}{l}2 \cdot 7 \\
3 \cdot 0\end{array}$ \\
\hline & $19 \underset{\mathbf{E}}{\mathbf{R}}$ & $\begin{array}{l}210 \\
370\end{array}$ & $\begin{array}{r}96 \\
108\end{array}$ & $\begin{array}{l}5 \cdot 2 \\
5 \cdot 6\end{array}$ & $\begin{array}{l}3 \cdot 75 \\
5 \cdot 1\end{array}$ & $\begin{array}{l}26 \\
36\end{array}$ & $\begin{array}{l}55 / 25 / 35 \\
70 / 30 / 45\end{array}$ & $60 / 7$ & $\begin{array}{l}115 / 55 / 70 \\
150 / 90 / 105\end{array}$ & $\begin{array}{r}7 \cdot 0 \\
11 \cdot 0\end{array}$ & $\begin{array}{l}22 \\
28\end{array}$ & $\begin{array}{l}3.3 \\
3 \cdot 0\end{array}$ \\
\hline & $20 \underset{\mathrm{E}}{\mathrm{R}}$ & $\begin{array}{l}190 \\
360\end{array}$ & $\begin{array}{l}70 \\
78\end{array}$ & $\begin{array}{l}5 \cdot 9 \\
8 \cdot 6\end{array}$ & $\begin{array}{l}3 \cdot 3 \\
4 \cdot 2\end{array}$ & $\begin{array}{l}22 \\
32\end{array}$ & $\begin{array}{l}60 / 20 / 35 \\
70 / 20 / 45\end{array}$ & $\underline{60 / 12}$ & $\begin{array}{l}125 / 50 / 80 \\
160 / 60 / 85\end{array}$ & $\begin{array}{r}7 \cdot 0 \\
16.5\end{array}$ & $\begin{array}{l}20 \\
25\end{array}$ & $\begin{array}{l}3.7 \\
4.6\end{array}$ \\
\hline & $21 \underset{\mathrm{E}}{\mathrm{R}}$ & $\begin{array}{l}250 \\
500\end{array}$ & $\begin{array}{r}72 \\
125\end{array}$ & $\begin{array}{r}7 \cdot 1 \\
13 \cdot 6\end{array}$ & $\begin{array}{l}3.5 \\
3.7\end{array}$ & 22 & $\begin{array}{l}65 / 35 / 45 \\
74 / 40 / 52\end{array}$ & 65/7 & $\begin{array}{l}140 / 75 / 90 \\
170 / 90 / 110\end{array}$ & $\begin{array}{r}7 \cdot 5 \\
23 \cdot 6\end{array}$ & $\begin{array}{l}19 \\
30\end{array}$ & $\begin{array}{l}3 \cdot 0 \\
4 \cdot 7\end{array}$ \\
\hline & $22 \underset{\mathrm{E}}{\mathrm{R}}$ & $\begin{array}{l}250 \\
460\end{array}$ & $\begin{array}{l}114 \\
125\end{array}$ & $\begin{array}{l}6.2 \\
6.5\end{array}$ & $\begin{array}{l}4 \cdot 0 \\
7 \cdot 0\end{array}$ & $\begin{array}{l}39 \\
50\end{array}$ & $\begin{array}{l}90 / 55 / 65 \\
115 / 65 / 85\end{array}$ & $90 / 15$ & $130 / 85 / 100$ & $\begin{array}{l}10 \cdot 6 \\
22 \cdot 5\end{array}$ & $\begin{array}{l}21 \\
41\end{array}$ & $\begin{array}{l}4 \cdot 3 \\
4 \cdot 9\end{array}$ \\
\hline & $23 \underset{E}{R}$ & $\begin{array}{l}195 \\
365\end{array}$ & $\begin{array}{r}90 \\
120\end{array}$ & $\begin{array}{l}5.9 \\
7.9\end{array}$ & $\begin{array}{l}3 \cdot 3 \\
4 \cdot 6\end{array}$ & $\begin{array}{l}20 \\
32\end{array}$ & $\begin{array}{r}95 / 48 / 62 \\
125 / 65 / 82\end{array}$ & $\underline{100 / 14}$ & $\begin{array}{l}215 / 129 / 155 \\
240 / 130 / 180\end{array}$ & $\begin{array}{r}6 \cdot 6 \\
15 \cdot 2\end{array}$ & $\begin{array}{l}15 \\
23\end{array}$ & $\begin{array}{l}3 \cdot 4 \\
4 \cdot 2\end{array}$ \\
\hline & $24 \underset{\mathbf{E}}{\mathbf{R}}$ & $\begin{array}{l}200 \\
450\end{array}$ & $\begin{array}{r}68 \\
150\end{array}$ & $\begin{array}{l}3 \cdot 8 \\
7 \cdot 6\end{array}$ & $\begin{array}{l}5 \cdot 2 \\
5 \cdot 9\end{array}$ & $\begin{array}{l}32 \\
55\end{array}$ & $\begin{array}{l}72 / 25 / 55 \\
85 / 40 / 65\end{array}$ & $80 / 8$ & $100 / 50 / 70$ & $\begin{array}{r}5 \cdot 9 \\
23 \cdot 8\end{array}$ & $\begin{array}{l}18 \\
18\end{array}$ & $\begin{array}{l}2 \cdot 6 \\
5 \cdot 3\end{array}$ \\
\hline & $25 \underset{\mathbf{E}}{\mathbf{R}}$ & $\begin{array}{l}215 \\
535\end{array}$ & $\begin{array}{l}54 \\
86\end{array}$ & $\begin{array}{l}4 \cdot 2 \\
6 \cdot 6\end{array}$ & $\begin{array}{l}5 \cdot 2 \\
7 \cdot 8\end{array}$ & $\begin{array}{l}13 \\
29\end{array}$ & $\begin{array}{l}28 / 9 / 16 \\
42 / 12 / 20\end{array}$ & $28 / 8$ & $\begin{array}{l}125 / 62 / 80 \\
150 / 75 / 95\end{array}$ & $\begin{array}{r}5 \cdot 5 \\
19 \cdot 8\end{array}$ & $\begin{array}{l}10 \\
20\end{array}$ & $\begin{array}{l}2.6 \\
3 \cdot 7\end{array}$ \\
\hline & $26 \mathbf{R}$ & 190 & 66 & 4.9 & $3 \cdot 8$ & 16 & $31 / 16 / 21$ & $32 / 9$ & $110 / 60 / 82$ & $5 \cdot 5$ & 14 & $2 \cdot 9$ \\
\hline
\end{tabular}


TABLE II-continued

\begin{tabular}{|c|c|c|c|c|c|c|c|c|c|c|c|c|}
\hline \multirow[b]{2}{*}{ Group } & \multirow[b]{2}{*}{$\begin{array}{l}\text { Case } \\
\text { No. }\end{array}$} & \multirow{2}{*}{$\begin{array}{c}\dot{\mathrm{v}}_{2} \\
\text { (ml./min. } \\
\text { STPDD }\end{array}$} & \multirow[b]{2}{*}{$\begin{array}{c}\text { Heart } \\
\text { rate }\end{array}$} & \multirow{2}{*}{$\begin{array}{c}\begin{array}{c}\text { A-V } \\
\text { diff. } \\
\text { (vol. \%) }\end{array} \\
\end{array}$} & \multirow[b]{2}{*}{$\begin{array}{c}\mathrm{CO} \\
\text { (1./min.) } \\
\end{array}$} & \multicolumn{4}{|c|}{ Pressures $(\mathrm{mm} . \mathbf{H g}$ ) } & \multirow{2}{*}{$\begin{array}{c}\dot{v} \\
\text { (1./min. } \\
\text { BTPS }\end{array}$} & \multirow[b]{2}{*}{$\begin{array}{c}\text { Resp. } \\
\text { rate }\end{array}$} & \multirow[b]{2}{*}{ VE } \\
\hline & & & & & & $\begin{array}{c}\text { PCV } \\
\text { (mean) }\end{array}$ & $\begin{array}{c}\mathrm{PA} \\
\mathrm{s} / \mathrm{d} / \text { mean }\end{array}$ & $\begin{array}{l}\mathrm{RV} \\
\text { s/d }\end{array}$ & $\begin{array}{c}\text { Syst. } \\
\text { s/d/mean }\end{array}$ & & & \\
\hline \multirow[t]{12}{*}{$\begin{array}{l}\text { III: Pulmo- } \\
\text { nary stenosis }\end{array}$} & $29 \underset{\mathrm{E}}{\mathrm{R}}$ & $\begin{array}{l}250 \\
760\end{array}$ & $\begin{array}{l}120 \\
188\end{array}$ & $\begin{array}{l}6 \cdot 2 \\
11 \cdot 7\end{array}$ & $\begin{array}{l}4 \cdot 0 \\
6.5\end{array}$ & $\overline{-}$ & $\begin{array}{l}40 / 20 / 25 \\
40 / 20 / 25\end{array}$ & $210 / 26$ & $\begin{array}{l}145 / 90 / 100 \\
150 / 100 / 110\end{array}$ & $\begin{array}{r}6.3 \\
22 \cdot 4\end{array}$ & $\begin{array}{l}16 \\
35\end{array}$ & $\begin{array}{l}2 \cdot 5 \\
3 \cdot 0\end{array}$ \\
\hline & $\begin{array}{r}30 \mathrm{R} \\
\mathrm{E}\end{array}$ & $\begin{array}{l}175 \\
450\end{array}$ & $\begin{array}{r}80 \\
120\end{array}$ & $\begin{array}{l}4 \cdot 5 \\
6 \cdot 0\end{array}$ & $\begin{array}{l}3.9 \\
7.5\end{array}$ & 9 & $\begin{array}{l}23 / 8 / 15 \\
23 / 8 / 15\end{array}$ & $\begin{array}{l}35 / 4 \\
65 / 3\end{array}$ & $\begin{array}{r}95 / 65 / 70 \\
100 / 67 / 80\end{array}$ & $\begin{array}{r}3 \cdot 3 \\
11 \cdot 1\end{array}$ & $=$ & $\begin{array}{l}1.9 \\
2.7\end{array}$ \\
\hline & $31 \underset{\mathrm{E}}{\mathrm{R}}$ & 150 & $=$ & $=$ & 二 & $\bar{z}$ & 二 & $115 / 10$ & 二 & 7.9 & 24 & $5 \cdot 3$ \\
\hline & $32 \underset{\mathrm{E}}{\mathrm{R}}$ & $\begin{array}{l}225 \\
560\end{array}$ & $\begin{array}{r}75 \\
104\end{array}$ & $\begin{array}{l}4.8 \\
8.5\end{array}$ & $\begin{array}{l}4 \cdot 6 \\
6 \cdot 6\end{array}$ & 9 & $\begin{array}{l}20 / 10 / 13 \\
20 / 10 / 13\end{array}$ & $\begin{array}{r}70 / 9 \\
100 / 0\end{array}$ & $\begin{array}{l}125 / 75 / 105 \\
140 / 68 / 95\end{array}$ & $\begin{array}{r}5 \cdot 7 \\
26 \cdot 2\end{array}$ & $\begin{array}{l}15 \\
22\end{array}$ & $\begin{array}{l}2 \cdot 5 \\
4 \cdot 6\end{array}$ \\
\hline & $\begin{array}{r}33 \mathrm{R} \\
\mathrm{E}\end{array}$ & $\begin{array}{l}196 \\
658\end{array}$ & $\begin{array}{r}80 \\
150\end{array}$ & $\begin{array}{l}4 \cdot 8 \\
8 \cdot 2\end{array}$ & $\begin{array}{l}4 \cdot 1 \\
8 \cdot 2\end{array}$ & $\underline{7}$ & $\begin{array}{l}16 / 8 / 11 \\
17 / 8 / 10\end{array}$ & $\begin{array}{l}55 / 12 \\
130 / 20\end{array}$ & $\begin{array}{l}105 / 65 / 83 \\
162 / 87 /-\end{array}$ & $\begin{array}{r}5 \cdot 2 \\
22 \cdot 2\end{array}$ & $\begin{array}{l}16 \\
25\end{array}$ & $\begin{array}{l}2 \cdot 7 \\
3 \cdot 4\end{array}$ \\
\hline & $\begin{array}{r}34 \underset{\mathrm{E}}{\mathrm{R}} \\
\mathrm{r}\end{array}$ & $\begin{array}{l}265 \\
695\end{array}$ & $\begin{array}{l}65 \\
94\end{array}$ & $\begin{array}{l}4 \cdot 0 \\
7.5\end{array}$ & $\begin{array}{l}6 \cdot 6 \\
9 \cdot 3\end{array}$ & $\underline{7}$ & $\begin{array}{l}25 / 10 / 13 \\
25 / 12 / 17\end{array}$ & $\begin{array}{l}50 / 10 \\
50 / 10\end{array}$ & $\begin{array}{l}110 / 60 / 70 \\
130 / 75 / 85\end{array}$ & $\begin{array}{r}7 \cdot 0 \\
13 \cdot 5\end{array}$ & $\begin{array}{l}14 \\
15\end{array}$ & $\begin{array}{l}2 \cdot 6 \\
2 \cdot 0\end{array}$ \\
\hline & $\begin{array}{r}35 \\
\mathbf{E}\end{array}$ & $\begin{array}{l}325 \\
700\end{array}$ & $\begin{array}{l}60 \\
92\end{array}$ & $\begin{array}{l}4 \cdot 6 \\
9 \cdot 0\end{array}$ & $\begin{array}{l}7.0 \\
7.8\end{array}$ & $\underline{-}$ & $15 / 8 / 12$ & $\begin{array}{l}125 / 10 \\
150 / 10\end{array}$ & $\begin{array}{l}125 / 70 / 85 \\
135 / 70 / 88\end{array}$ & $\begin{array}{r}7 \cdot 7 \\
24 \cdot 2\end{array}$ & $\begin{array}{l}14 \\
18\end{array}$ & $\begin{array}{l}2.4 \\
3.5\end{array}$ \\
\hline & $36 \underset{E}{\mathrm{R}}$ & $\begin{array}{l}270 \\
750\end{array}$ & $\begin{array}{r}70 \\
125\end{array}$ & $\begin{array}{r}4 \cdot 8 \\
10 \cdot 0\end{array}$ & $\begin{array}{l}5 \cdot 6 \\
7 \cdot 1\end{array}$ & 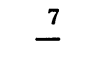 & $\begin{array}{l}20 / 10 / 12 \\
28 / 15 / 20\end{array}$ & $\begin{array}{l}40 / 5 \\
60 / 5\end{array}$ & $\begin{array}{l}115 / 60 / 75 \\
140 / 70 / 80\end{array}$ & $\begin{array}{r}5 \cdot 0 \\
19 \cdot 3\end{array}$ & $\begin{array}{r}99 \\
16\end{array}$ & $\begin{array}{l}1.9 \\
2.6\end{array}$ \\
\hline & $\begin{array}{r}37 \mathrm{R} \\
\mathrm{E}\end{array}$ & $\begin{array}{r}240 \\
1060\end{array}$ & $\begin{array}{r}68 \\
115\end{array}$ & $\begin{array}{l}5.7 \\
8.9\end{array}$ & $\begin{array}{r}4.2 \\
11.8\end{array}$ & $\underline{10}$ & $\begin{array}{l}22 / 10 / 16 \\
22 / 10 / 17\end{array}$ & $\begin{array}{r}85 / 10 \\
100 / 10\end{array}$ & $\begin{array}{l}125 / 65 / 80 \\
140 / 75 / 80\end{array}$ & $\begin{array}{r}6 \cdot 2 \\
28 \cdot 0\end{array}$ & $\begin{array}{l}10 \\
30\end{array}$ & $\begin{array}{l}2 \cdot 6 \\
2 \cdot 6\end{array}$ \\
\hline & $\begin{array}{r}38 \mathbf{R} \\
\mathbf{E}\end{array}$ & $\begin{array}{l}230 \\
540\end{array}$ & $\begin{array}{l}63 \\
94\end{array}$ & $\begin{array}{r}4 \cdot 8 \\
10 \cdot 2\end{array}$ & $\begin{array}{l}4 \cdot 8 \\
5 \cdot 3\end{array}$ & 9 & $\begin{array}{l}20 / 10 / 12 \\
30 / 15 / 18\end{array}$ & $\begin{array}{l}60 / 10 \\
70 / 10\end{array}$ & $\begin{array}{l}110 / 65 / 80 \\
150 / 75 / 100\end{array}$ & $\begin{array}{r}6 \cdot 2 \\
16 \cdot 2\end{array}$ & $\begin{array}{l}14 \\
21\end{array}$ & $\begin{array}{l}2 \cdot 7 \\
3.0\end{array}$ \\
\hline & $39 \underset{\mathrm{E}}{\mathrm{R}}$ & $\begin{array}{l}200 \\
375\end{array}$ & $\begin{array}{l}70 \\
78\end{array}$ & $\begin{array}{l}5 \cdot 7 \\
9 \cdot 1\end{array}$ & $\begin{array}{l}3 \cdot 5 \\
4 \cdot 1\end{array}$ & 二 & $\begin{array}{l}28 / 18 / 22 \\
32 / 16 / 22\end{array}$ & $\begin{array}{l}90 / 7 \\
100 / 7\end{array}$ & $130 / 65 / 85$ & $\begin{array}{r}6 \cdot 2 \\
16 \cdot 5\end{array}$ & $\begin{array}{l}14 \\
25\end{array}$ & $\begin{array}{l}3 \cdot 1 \\
4 \cdot 1\end{array}$ \\
\hline & $40 \mathrm{R}$ & 285 & 70 & $8 \cdot 8$ & $3 \cdot 2$ & 10 & $22 / 17 / 18$ & $258 / 27$ & - & $5 \cdot 8$ & 23 & $2 \cdot 2$ \\
\hline
\end{tabular}

$\dot{V}_{2}$, oxygen uptake; A-V diff., arteriovenous oxygen differences; CO, cardiac output; PCV, pulmonary capillary venous (wedge); PA, pulmonary arterial; RV, right ventricle; Syst., systemic; V, ventilation; VE, ventilatory equivalent; STPD, standard temperature and pressure, dry $\left(0^{\circ} \mathrm{C}\right.$. and $760 \mathrm{~mm}$. Hg); BTPS, body temperature and pressure saturated with water vapour; $R$, rest; $E$, exercise.

at rest and on effort in the subjects studied during mild supine exercise on the catheter table.

The changes with exercise in the ventilation and the main components of arterial blood chemistry are illustrated in Fig. 1. The responses to exercise of cardiac output and mixed venous blood composition are illustrated in Fig. 2. The first points in the figure represent the resting values, the second those in the third-to-fourth minute and the last those at about the tenth minute of exercise. In the first column of the figures the corresponding findings in the few normal subjects used for comparison are shown.

In Fig. 1 it is seen, as anticipated, that the ventilatory response to exercise is greater in the patients with cardiac lesions, particularly those with mitral disease. The systemic arterial oxygen saturation was lower than normal in some patients in all three groups. Only 3, however, showed an oxygen saturation below 90 per cent on effort, one of those (Case 16) being the patient who developed pulmonary odema, another having mitral valve disease with additional lung disease (Case 23), the third (Case 33) being a patient with pulmonary stenosis and a small shunt through a patent foramen ovale.

The levels of the arterial $\mathrm{PCO}_{2}$ showed no marked differences between the groups, and certainly no tendency to rise in the group with the greatest increment in ventilation. In most cases the highest ventilatory response was associated with low values of $\mathrm{PCO}_{2}$ as shown in Fig. 3. Only three patients had an exercise $\mathrm{PCO}_{2}$ higher than $45 \mathrm{~mm}$. $\mathrm{Hg}$ (Cases 11, 14, and 23). The findings in those 3 suggested the presence of lung disease, and this was confirmed by lung function studies in the 2 patients in whom these were performed; both showed a restrictive ventilatory defect and evidence of gaseous maldistribution in the lung.

The arterial $p \mathrm{H}$ also showed no apparent correlation with the ventilation, and patients with higher ventilation were usually the more alkalotic ones (Fig. 4).

The arterial lactate is plotted in Fig. 5 against the excess ventilation. The patients with greatest deviation of ventilation from normal have higher lactates, but the relationship is not significant $(\mathrm{p}>0 \cdot 1)$. We noted, moreover, that in cases with a ventilation as much as 100 per cent above the normal for the exercise load, the lactate levels lay within the wide normal range. This will be discussed later.

Finally in Fig. 6 the relationship is shown between 
TABLE III

SIMULTANEOUS ARTERIAL AND MIXED VENOUS BLOOD COMPOSITION AT REST AND DURING STEADY-STATE MILD SUPINE EXERCISE

\begin{tabular}{|c|c|c|c|c|c|c|c|c|c|c|c|c|}
\hline \multirow[b]{2}{*}{ Group } & \multirow{2}{*}{\multicolumn{2}{|c|}{$\begin{array}{l}\text { Case } \\
\text { No. }\end{array}$}} & \multicolumn{6}{|c|}{ Arterial Blood } & \multicolumn{4}{|c|}{ Venous Blood } \\
\hline & & & $\mathrm{SAO}_{2}$ & $p \mathrm{H}$ & $\underset{(\mathrm{mm} . \mathrm{Hg})}{\mathrm{PcO}_{2}}$ & $\begin{array}{c}\text { Bicarb. } \\
\text { (mEq/1.) }\end{array}$ & $\underset{(\mathrm{mM} / \mathrm{l} .)}{\text { Lact. }}$ & $\underset{(\mathrm{mM} / \mathrm{l} .)}{\mathrm{Pyr}}$ & $\mathrm{Sv̄o}_{2}$ & $p \mathrm{H}$ & $\underset{(\mathrm{mm} .}{\mathrm{Pco}_{\mathbf{T}}}$ & $\underset{(\mathrm{mEq} / \mathrm{l} .)}{\text { Bicarb. }}$ \\
\hline \multirow[t]{6}{*}{ Normal } & 1 & $\underset{\mathbf{E}}{\mathbf{R}}$ & $\begin{array}{l}96 \cdot 4 \\
97 \cdot 1 \\
94 \cdot 7\end{array}$ & $\begin{array}{l}7 \cdot 500 \\
7 \cdot 470 \\
7 \cdot 410\end{array}$ & $\begin{array}{l}27 \cdot 5 \\
30 \cdot 0 \\
36 \cdot 0\end{array}$ & $\begin{array}{l}25 \cdot 5 \\
24 \cdot 0 \\
23 \cdot 0\end{array}$ & $\begin{array}{l}0.98 \\
1.95 \\
2.15\end{array}$ & $\begin{array}{l}0.09 \\
0.16 \\
0.18\end{array}$ & $\begin{array}{l}78 \cdot 4 \\
54 \cdot 2 \\
62 \cdot 1\end{array}$ & $\begin{array}{l}7 \cdot 470 \\
7 \cdot 380 \\
7 \cdot 389\end{array}$ & $\begin{array}{l}31 \cdot 0 \\
46 \cdot 0 \\
42 \cdot 0\end{array}$ & $\begin{array}{l}24 \cdot 0 \\
23 \cdot 5 \\
22 \cdot 0\end{array}$ \\
\hline & 2 & $\underset{\mathbf{E}}{\mathbf{R}}$ & $\begin{array}{l}98.7 \\
28 \cdot 2 \\
95 \cdot 5\end{array}$ & $\begin{array}{l}7.435 \\
7.405 \\
7.395\end{array}$ & $\begin{array}{l}36.0 \\
36 \cdot 0 \\
35.0\end{array}$ & $\begin{array}{l}24 \cdot 5 \\
23 \cdot 0 \\
22 \cdot 0\end{array}$ & $\begin{array}{l}0.91 \\
1.95 \\
2.02\end{array}$ & $\begin{array}{l}0.06 \\
0 \cdot 13 \\
0 \cdot 17\end{array}$ & $\begin{array}{l}79.7 \\
55.0 \\
51.5\end{array}$ & $\begin{array}{l}7.410 \\
7.345 \\
7.335\end{array}$ & $\begin{array}{l}43 \cdot 0 \\
41.0 \\
45.0\end{array}$ & $\begin{array}{l}25.0 \\
20.5 \\
20.5\end{array}$ \\
\hline & 3 & $\begin{array}{l}\mathbf{R} \\
\mathbf{E}\end{array}$ & $\begin{array}{l}96.5 \\
98.6 \\
96.5\end{array}$ & $\begin{array}{l}7 \cdot 400 \\
7 \cdot 400 \\
7 \cdot 400\end{array}$ & $\begin{array}{l}41.0 \\
38.0 \\
39.0\end{array}$ & $\begin{array}{l}24 \cdot 3 \\
23 \cdot 3 \\
23 \cdot 5\end{array}$ & $\begin{array}{l}1.04 \\
2 \cdot 34 \\
2.08\end{array}$ & $\begin{array}{l}0 \cdot 11 \\
0 \cdot 14 \\
0 \cdot 14\end{array}$ & $\begin{array}{l}81.5 \\
63.9 \\
70.5\end{array}$ & $\begin{array}{l}7 \cdot 370 \\
7 \cdot 365 \\
7 \cdot 360\end{array}$ & $\begin{array}{l}47 \cdot 0 \\
46 \cdot 0 \\
49 \cdot 0\end{array}$ & $\begin{array}{l}24 \cdot 0 \\
23 \cdot 0 \\
23 \cdot 5\end{array}$ \\
\hline & 4 & $\underset{\mathrm{E}}{\mathrm{R}}$ & $\begin{array}{l}98 \cdot 6 \\
98 \cdot 6 \\
98 \cdot 6\end{array}$ & $\begin{array}{l}7 \cdot 375 \\
7 \cdot 370 \\
7 \cdot 380\end{array}$ & $\begin{array}{l}44 \cdot 0 \\
44 \cdot 0 \\
45 \cdot 0\end{array}$ & $\begin{array}{l}23 \cdot 5 \\
23 \cdot 5 \\
24 \cdot 5\end{array}$ & $\begin{array}{l}1 \cdot 18 \\
2 \cdot 60 \\
2 \cdot 08\end{array}$ & $\begin{array}{l}0 \cdot 12 \\
0 \cdot 18 \\
0 \cdot 18\end{array}$ & $\begin{array}{l}80 \cdot 0 \\
78 \cdot 0 \\
78 \cdot 0\end{array}$ & $\begin{array}{l}7 \cdot 340 \\
7 \cdot 335 \\
7 \cdot 360\end{array}$ & $\begin{array}{l}52 \cdot 0 \\
54 \cdot 0 \\
51 \cdot 0\end{array}$ & $\begin{array}{l}24 \cdot 0 \\
23 \cdot 5 \\
24 \cdot 5\end{array}$ \\
\hline & 5 & $\underset{R}{R}$ & $98 \cdot 0$ & $7 \cdot 455$ & 36.5 & - & 0.75 & 0.07 & $74 \cdot 6$ & $7 \cdot 415$ & 39.5 & 23.0 \\
\hline & & & $9 \overline{98.0}$ & $\overline{7 \cdot 415}$ & $4 \overline{0 \cdot 0}$ & 二 & $\overline{1.04}$ & $0 . \overline{09}$ & 二 & $7 \cdot \overline{4} 10$ & 二 & 二 \\
\hline \multirow{10}{*}{ 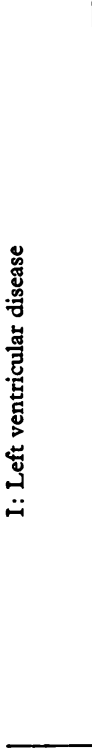 } & 6 & $\begin{array}{l}\mathbf{R} \\
\mathbf{E}\end{array}$ & $\begin{array}{l}98 \cdot 4 \\
96 \cdot 2 \\
95 \cdot 6\end{array}$ & $\begin{array}{l}7 \cdot 400 \\
7 \cdot 365 \\
7 \cdot 375\end{array}$ & $\begin{array}{l}44.5 \\
48 \cdot 0 \\
42 \cdot 0\end{array}$ & $\begin{array}{l}25 \cdot 4 \\
25.0 \\
23.5\end{array}$ & $\begin{array}{l}0.46 \\
1.24 \\
1.89\end{array}$ & $\begin{array}{l}0 \cdot 10 \\
0 \cdot 18 \\
0 \cdot 18\end{array}$ & $\begin{array}{l}67 \cdot 5 \\
50 \cdot 4 \\
50 \cdot 5\end{array}$ & $\begin{array}{l}7 \cdot 390 \\
7 \cdot 350 \\
7 \cdot 335\end{array}$ & $\begin{array}{l}50 \cdot 0 \\
52.0 \\
54 \cdot 0\end{array}$ & $\begin{array}{l}25.4 \\
24.6 \\
20.5\end{array}$ \\
\hline & 7 & $\underset{\mathbf{E}}{\mathbf{R}}$ & $\begin{array}{l}97 \cdot 0 \\
95 \cdot 6 \\
97 \cdot 6\end{array}$ & $\begin{array}{l}7 \cdot 380 \\
7 \cdot 390 \\
7 \cdot 400\end{array}$ & $\begin{array}{l}32.5 \\
33.0 \\
33.0\end{array}$ & $\begin{array}{l}20 \cdot 0 \\
21 \cdot 5 \\
22 \cdot 0\end{array}$ & $\begin{array}{l}1 \cdot 30 \\
1 \cdot 30 \\
1 \cdot 47\end{array}$ & $\begin{array}{l}0.04 \\
0 \cdot 14 \\
0 \cdot 14\end{array}$ & $\begin{array}{l}78 \cdot 5 \\
68 \cdot 8 \\
71 \cdot 6\end{array}$ & $\begin{array}{l}7 \cdot 360 \\
7 \cdot 340 \\
7 \cdot 340\end{array}$ & $\begin{array}{l}40 \cdot 0 \\
48 \cdot 0 \\
46 \cdot 0\end{array}$ & $\begin{array}{l}22 \cdot 0 \\
22 \cdot 5 \\
22 \cdot 0\end{array}$ \\
\hline & 8 & $\underset{\mathbf{E}}{\mathbf{R}}$ & $\begin{array}{l}96 \cdot 4 \\
92 \cdot 4 \\
93 \cdot 7\end{array}$ & $\begin{array}{l}7 \cdot 425 \\
7 \cdot 395 \\
7 \cdot 400\end{array}$ & $\begin{array}{l}38.0 \\
39 \cdot 0 \\
38.0\end{array}$ & $\begin{array}{l}24 \cdot 5 \\
23.0 \\
23.0\end{array}$ & $\begin{array}{l}1 \cdot 30 \\
1 \cdot 82 \\
2 \cdot 21\end{array}$ & 二 & $\begin{array}{l}67 \cdot 5 \\
68 \cdot 8 \\
63 \cdot 4\end{array}$ & $\begin{array}{l}7 \cdot 410 \\
7 \cdot 370 \\
7 \cdot 390\end{array}$ & $\begin{array}{l}41 \cdot 0 \\
40 \cdot 5 \\
43 \cdot 0\end{array}$ & $\begin{array}{l}24 \cdot 0 \\
21 \cdot 5 \\
24 \cdot 0\end{array}$ \\
\hline & 9 & $\underset{\mathbf{E}}{\mathbf{R}}$ & $\begin{array}{l}97 \cdot 5 \\
98 \cdot 0 \\
99 \cdot 2\end{array}$ & $\begin{array}{l}7 \cdot 390 \\
7 \cdot 360 \\
7 \cdot 385\end{array}$ & $\begin{array}{l}35.0 \\
38.0 \\
36.5\end{array}$ & $\begin{array}{l}21.5 \\
21.0 \\
21.5\end{array}$ & $\begin{array}{l}0.52 \\
1.04 \\
1 \cdot 17\end{array}$ & 二 & $\begin{array}{l}70 \cdot 0 \\
53 \cdot 3 \\
50 \cdot 4\end{array}$ & $\begin{array}{l}7 \cdot 375 \\
7 \cdot 340 \\
7 \cdot 350\end{array}$ & $\begin{array}{l}41 \cdot 0 \\
48 \cdot 0 \\
45 \cdot 0\end{array}$ & $\begin{array}{l}22 \cdot 0 \\
22 \cdot 0 \\
21 \cdot 5\end{array}$ \\
\hline & 10 & $\underset{\mathbf{E}}{\mathbf{R}}$ & $\begin{array}{l}91.5 \\
95.0 \\
95.8\end{array}$ & $\begin{array}{l}7 \cdot 405 \\
7 \cdot 410 \\
7 \cdot 405\end{array}$ & $\begin{array}{l}36.5 \\
35.0 \\
36.5\end{array}$ & $\begin{array}{l}23 \cdot 0 \\
23 \cdot 0 \\
23.0\end{array}$ & $\begin{array}{l}0.97 \\
1.47 \\
1.36\end{array}$ & $\begin{array}{l}0.05 \\
0.07 \\
-\end{array}$ & $\begin{array}{l}65 \cdot 8 \\
64 \cdot 1 \\
62 \cdot 9\end{array}$ & $\begin{array}{l}7 \cdot 395 \\
7 \cdot 360 \\
7 \cdot 395\end{array}$ & $\begin{array}{l}40 \cdot 0 \\
46 \cdot 0 \\
39 \cdot 5\end{array}$ & $\begin{array}{l}23.0 \\
22.5 \\
22.5\end{array}$ \\
\hline & 11 & $\underset{\mathbf{E}}{\mathbf{R}}$ & $\stackrel{93 \cdot 3}{-}$ & $7 \cdot \underline{782}$ & $48 \cdot 0$ & 25.0 & $1 \cdot 17$ & $0 \cdot 13$ & $68 \cdot 3$ & $7 \cdot 315$ & $51 \cdot 0$ & $21 \cdot 0$ \\
\hline & & & 92.5 & $7 \cdot 351$ & $4 \overline{46.5}$ & $2 \overline{23.0}$ & $\overline{1.47}$ & $0 \cdot \overline{13}$ & 63.0 & $7 \cdot \overline{315}$ & $\overline{49 \cdot 0}$ & $2 \overline{21 \cdot 0}$ \\
\hline & 12 & $\underset{\mathbf{E}}{\mathbf{R}}$ & $\begin{array}{l}95 \cdot 5 \\
94 \cdot 2 \\
96 \cdot 2\end{array}$ & $\begin{array}{l}7 \cdot 420 \\
7 \cdot 410 \\
7 \cdot 420\end{array}$ & $\begin{array}{l}40 \cdot 0 \\
40 \cdot 0 \\
37 \cdot 0\end{array}$ & $\begin{array}{l}25 \cdot 0 \\
24 \cdot 5 \\
24 \cdot 0\end{array}$ & $\begin{array}{l}0.58 \\
1.69 \\
1.43\end{array}$ & $\begin{array}{l}0.07 \\
0.11 \\
0.13\end{array}$ & $\begin{array}{l}69.4 \\
49.9 \\
53.0\end{array}$ & 二 & $\bar{z}$ & 二 \\
\hline & 13 & $\underset{\mathbf{E}}{\mathbf{R}}$ & $\begin{array}{l}96 \cdot 2 \\
97 \cdot 4 \\
95 \cdot 9\end{array}$ & $\begin{array}{l}\mathbf{7} \cdot 380 \\
7 \cdot 370 \\
7 \cdot 395\end{array}$ & $\begin{array}{l}41.0 \\
43.5 \\
38.5\end{array}$ & $\begin{array}{l}23 \cdot 0 \\
23 \cdot 0 \\
23 \cdot 0\end{array}$ & $\begin{array}{l}0.58 \\
0.97 \\
0.65\end{array}$ & $\begin{array}{l}0.04 \\
0.07 \\
0.06\end{array}$ & $\begin{array}{l}74 \cdot 4 \\
62 \cdot 7 \\
62 \cdot 8\end{array}$ & $\begin{array}{l}7 \cdot 350 \\
7 \cdot 325 \\
7 \cdot 355\end{array}$ & $\begin{array}{l}44.0 \\
50.5 \\
44.0\end{array}$ & $\begin{array}{l}22 \cdot 0 \\
22 \cdot 0 \\
22 \cdot 0\end{array}$ \\
\hline & 14 & $\underset{\mathbf{E}}{\mathbf{R}}$ & $\begin{array}{l}91 \cdot 2 \\
90 \cdot 8 \\
94 \cdot 6\end{array}$ & $\begin{array}{l}7 \cdot 420 \\
7 \cdot 390 \\
7 \cdot 360\end{array}$ & $\begin{array}{l}49.0 \\
54.0 \\
55.0\end{array}$ & $\begin{array}{l}28 \cdot 0 \\
28 \cdot 0 \\
26 \cdot 0\end{array}$ & $\begin{array}{l}0.65 \\
1.30 \\
1.37\end{array}$ & $\begin{array}{l}0.05 \\
0.06 \\
0.08\end{array}$ & $\begin{array}{l}61.0 \\
53.0 \\
48.0\end{array}$ & $\begin{array}{l}7 \cdot 390 \\
7 \cdot 360 \\
7 \cdot 370\end{array}$ & $\begin{array}{l}60.0 \\
64.0 \\
59.0\end{array}$ & $\begin{array}{l}29 \cdot 0 \\
27 \cdot 0 \\
27 \cdot 5\end{array}$ \\
\hline \multirow{6}{*}{ 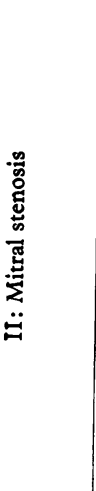 } & & $\underset{\mathbf{E}}{\mathbf{R}}$ & $\begin{array}{l}95 \cdot 5 \\
96 \cdot 3 \\
97 \cdot 7\end{array}$ & $\begin{array}{l}7 \cdot 380 \\
7 \cdot 370 \\
7 \cdot 350\end{array}$ & $\begin{array}{l}40.5 \\
41 \cdot 5 \\
44 \cdot 0\end{array}$ & $\begin{array}{l}23.0 \\
23.5 \\
22.5\end{array}$ & $\begin{array}{l}0.84 \\
1.56 \\
1.36\end{array}$ & $\begin{array}{l}0.07 \\
0.12 \\
0.12\end{array}$ & $\begin{array}{l}78 \cdot 2 \\
72 \cdot 5 \\
73 \cdot 7\end{array}$ & $\begin{array}{l}7 \cdot 360 \\
7 \cdot 355 \\
7 \cdot 355\end{array}$ & $\begin{array}{l}50.0 \\
46.0 \\
47.0\end{array}$ & $\begin{array}{l}24 \cdot 0 \\
22 \cdot 0 \\
23 \cdot 0\end{array}$ \\
\hline & 16 & $\begin{array}{l}\mathbf{R} \\
\mathbf{E}\end{array}$ & $\begin{array}{l}90 \cdot 1 \\
90 \cdot 9 \\
84 \cdot 8\end{array}$ & $\begin{array}{l}7 \cdot 450 \\
7 \cdot 390 \\
7 \cdot 395\end{array}$ & $\begin{array}{l}28.0 \\
34.0 \\
31 \cdot 5\end{array}$ & $\begin{array}{l}22.0 \\
21.0 \\
20.5\end{array}$ & $\begin{array}{l}0.91 \\
2 \cdot 34 \\
3 \cdot 58\end{array}$ & $\begin{array}{l}0 \cdot 11 \\
0 \cdot 15 \\
0 \cdot 19\end{array}$ & $\begin{array}{l}57 \cdot 1 \\
31 \cdot 3 \\
32 \cdot 0\end{array}$ & $\begin{array}{l}7 \cdot 440 \\
7 \cdot 350 \\
7 \cdot 350\end{array}$ & $\begin{array}{l}31 \cdot 0 \\
45 \cdot 0 \\
44 \cdot 0\end{array}$ & $\begin{array}{l}22 \cdot 0 \\
21 \cdot 0 \\
20 \cdot 5\end{array}$ \\
\hline & 17 & $\begin{array}{l}\mathbf{R} \\
\mathbf{E}\end{array}$ & $\begin{array}{l}93 \cdot 2 \\
91 \cdot 8 \\
94 \cdot 3\end{array}$ & $\begin{array}{l}7 \cdot 405 \\
7 \cdot 380 \\
7 \cdot 395\end{array}$ & $\begin{array}{l}35 \cdot 5 \\
42 \cdot 0 \\
38 \cdot 0\end{array}$ & $\begin{array}{l}23 \cdot 0 \\
23 \cdot 5 \\
23 \cdot 0\end{array}$ & $\begin{array}{l}0.65 \\
0.98 \\
0.85\end{array}$ & $\begin{array}{l}0.07 \\
0 \cdot 10 \\
0.09\end{array}$ & $\begin{array}{l}64.5 \\
57.5 \\
57.5\end{array}$ & $\begin{array}{l}7 \cdot 360 \\
7 \cdot 350 \\
7 \cdot 350\end{array}$ & $\begin{array}{l}45 \cdot 0 \\
49 \cdot 0 \\
49 \cdot 0\end{array}$ & $\begin{array}{l}22 \cdot 5 \\
22 \cdot 5 \\
23 \cdot 0\end{array}$ \\
\hline & 18 & $\underset{\mathbf{E}}{\mathbf{R}}$ & $\begin{array}{l}93 \cdot 2 \\
93 \cdot 6 \\
93 \cdot 3\end{array}$ & $\begin{array}{l}7 \cdot 430 \\
7 \cdot 445 \\
7 \cdot 460\end{array}$ & $\begin{array}{l}49 \cdot 0 \\
42 \cdot 0 \\
35 \cdot 5\end{array}$ & $\begin{array}{l}29 \cdot 0 \\
27 \cdot 0 \\
25 \cdot 5\end{array}$ & $\begin{array}{l}1.36 \\
2.47 \\
2.99\end{array}$ & $\begin{array}{l}0.11 \\
0.13 \\
0.15\end{array}$ & $\begin{array}{l}60 \cdot 0 \\
42.6 \\
41 \cdot 0\end{array}$ & $\begin{array}{l}7 \cdot 395 \\
7 \cdot 375 \\
7 \cdot 370\end{array}$ & $\begin{array}{l}52.0 \\
55.0 \\
55.5\end{array}$ & $\begin{array}{l}26 \cdot 5 \\
25 \cdot 0 \\
25 \cdot 0\end{array}$ \\
\hline & & $\begin{array}{l}\mathrm{R} \\
\mathrm{E}\end{array}$ & $\begin{array}{l}95.8 \\
94.5 \\
98.0\end{array}$ & $\begin{array}{l}7 \cdot 385 \\
7 \cdot 405 \\
7 \cdot 465\end{array}$ & $\begin{array}{l}43.0 \\
40.0 \\
30.0\end{array}$ & $\begin{array}{l}24 \cdot 0 \\
24 \cdot 0 \\
24 \cdot 0\end{array}$ & $\begin{array}{l}0.52 \\
0.78 \\
1 \cdot 17\end{array}$ & $\begin{array}{l}0.06 \\
0 \cdot 10 \\
0 \cdot 11\end{array}$ & $\begin{array}{l}64.0 \\
56 \cdot 0 \\
56.0\end{array}$ & $\begin{array}{l}7 \cdot 365 \\
7 \cdot 345 \\
7 \cdot 385\end{array}$ & $\begin{array}{l}45 \cdot 0 \\
49 \cdot 0 \\
38 \cdot 5\end{array}$ & $\begin{array}{l}22 \cdot 0 \\
22 \cdot 0 \\
22 \cdot 0\end{array}$ \\
\hline & 20 & $\begin{array}{l}\mathbf{R} \\
\mathbf{E}\end{array}$ & $\begin{array}{l}96.0 \\
95.0 \\
94.4\end{array}$ & $\begin{array}{l}7 \cdot 425 \\
7 \cdot 425 \\
7 \cdot 420\end{array}$ & $\begin{array}{l}40 \cdot 0 \\
38 \cdot 5 \\
37 \cdot 0\end{array}$ & $\begin{array}{l}25 \cdot 5 \\
25 \cdot 0 \\
24 \cdot 0\end{array}$ & $\begin{array}{l}1.36 \\
1.82 \\
1.82\end{array}$ & $\begin{array}{l}0.08 \\
0 \cdot 10 \\
0 \cdot 12\end{array}$ & $\begin{array}{l}\mathbf{6 4} \cdot 5 \\
44 \cdot 4 \\
57 \cdot 9\end{array}$ & $\begin{array}{l}7 \cdot 415 \\
7 \cdot 370 \\
7 \cdot 400\end{array}$ & $\begin{array}{l}46 \cdot 0 \\
46 \cdot 0 \\
43 \cdot 0\end{array}$ & $\begin{array}{l}25 \cdot 5 \\
23 \cdot 0 \\
23 \cdot 0\end{array}$ \\
\hline
\end{tabular}


Ventilation and Blood Chemistry in Heart Disease

TABLE III-continued

\begin{tabular}{|c|c|c|c|c|c|c|c|c|c|c|c|c|}
\hline \multirow[b]{2}{*}{ Group } & \multirow{2}{*}{\multicolumn{2}{|c|}{$\begin{array}{l}\text { Case } \\
\text { No. }\end{array}$}} & \multicolumn{6}{|c|}{ Arterial Blood } & \multicolumn{4}{|c|}{ Venous Blood } \\
\hline & & & $\mathrm{SAO}_{2}$ & $p \mathrm{H}$ & $\begin{array}{c}\mathrm{Pco}_{2} \\
(\mathrm{~mm} . \mathrm{Hg})\end{array}$ & $\begin{array}{c}\text { Bicarb. } \\
(\mathrm{mEq} / \mathrm{l} .)\end{array}$ & $\begin{array}{c}\text { Lact. } \\
(\mathrm{mM} / 1 .)\end{array}$ & $\underset{(\mathrm{mM} / \mathrm{l} .)}{\operatorname{Pyr}}$ & $\mathrm{SV}_{\overline{\mathrm{O}}}{ }_{2}$ & $p \mathrm{H}$ & $\begin{array}{c}\mathrm{PcO}_{2} \\
(\mathrm{~mm} . \mathrm{Hg})\end{array}$ & $\begin{array}{c}\text { Bicarb. } \\
(\mathrm{mEq} / \mathrm{l} .)\end{array}$ \\
\hline \multirow{7}{*}{ 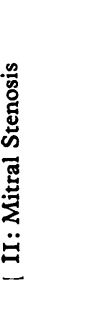 } & & & $\begin{array}{l}92 \cdot 8 \\
93.3\end{array}$ & $7 \cdot 405$ & 33.0 & $22 \cdot 0$ & 0.77 & 0.08 & $61 \cdot 2$ & $7 \cdot 390$ & $38 \cdot 0$ & 21.5 \\
\hline & & & 95.5 & $7 \cdot 395$ & $29 \cdot 0$ & $21 \cdot 0$ & $1 \cdot 48$ & $0 \cdot 10$ & $33 \cdot 5$ & $7 \cdot 325$ & $44 \cdot 0$ & $19 \cdot 0$ \\
\hline & 22 & $\begin{array}{l}\mathbf{R} \\
\mathbf{E}\end{array}$ & $\begin{array}{l}92 \cdot 0 \\
94.0\end{array}$ & $\begin{array}{l}7 \cdot 430 \\
7 \cdot 460\end{array}$ & $\begin{array}{l}27 \cdot 5 \\
26.5\end{array}$ & $\begin{array}{l}20.5 \\
21.5\end{array}$ & $\begin{array}{l}1 \cdot 17 \\
2 \cdot 08\end{array}$ & $\begin{array}{l}0.12 \\
0.13\end{array}$ & $\begin{array}{l}30 \cdot 0 \\
28.5\end{array}$ & $\begin{array}{l}7 \cdot 405 \\
7 \cdot 365\end{array}$ & $\begin{array}{l}37 \cdot 0 \\
46 \cdot 0\end{array}$ & $\begin{array}{l}22 \cdot 0 \\
23 \cdot 0\end{array}$ \\
\hline & & & & & & & & & & & & \\
\hline & 23 & $\underset{\mathbf{E}}{\mathbf{R}}$ & 90.5 & $7 \cdot 310$ & $\underline{49 \cdot 0}$ & 21.5 & $\begin{array}{l}1 \cdot 43 \\
2 \cdot 27\end{array}$ & $\begin{array}{l}0.06 \\
0.12\end{array}$ & $\begin{array}{l}62 \cdot 3 \\
51 \cdot 8\end{array}$ & $\begin{array}{l}7 \cdot 300 \\
7 \cdot 285\end{array}$ & $\begin{array}{l}59.0 \\
61.0\end{array}$ & $22 \cdot 3$ \\
\hline & & & $89 \cdot 6$ & $7 \cdot 300$ & $47 \cdot 0$ & $20 \cdot 0$ & $2 \cdot 08$ & $0 \cdot 12$ & $53 \cdot 8$ & $7 \cdot 265$ & $55 \cdot 0$ & $18 \cdot 5$ \\
\hline & 24 & $\underset{\mathbf{E}}{\mathbf{R}}$ & $\begin{array}{l}92 \cdot 7 \\
94 \cdot 4 \\
93 \cdot 6\end{array}$ & $\begin{array}{l}7 \cdot 445 \\
7 \cdot 430 \\
7 \cdot 475\end{array}$ & $\begin{array}{l}33.5 \\
34.0 \\
27.0\end{array}$ & $\begin{array}{l}24.0 \\
23.5 \\
22.5\end{array}$ & $\begin{array}{l}1.24 \\
3.57 \\
3.64\end{array}$ & $\frac{0.07}{0.18}$ & $\begin{array}{l}71 \cdot 4 \\
48 \cdot 2 \\
49 \cdot 6\end{array}$ & $\begin{array}{l}7 \cdot 415 \\
7 \cdot 360 \\
7 \cdot 375\end{array}$ & $\begin{array}{l}40 \cdot 0 \\
48 \cdot 0 \\
42 \cdot 0\end{array}$ & $\begin{array}{l}24 \cdot 0 \\
22 \cdot 5 \\
22 \cdot 0\end{array}$ \\
\hline \multirow{16}{*}{ 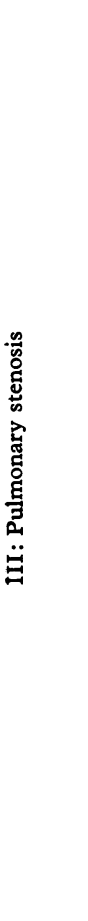 } & 29 & $\underset{\mathbf{E}}{\mathbf{R}}$ & $\begin{array}{r}97 \cdot 1 \\
97 \cdot 7 \\
100 \cdot 0\end{array}$ & $\begin{array}{l}7 \cdot 410 \\
7 \cdot 345 \\
7 \cdot 340\end{array}$ & $\begin{array}{l}34.0 \\
31 \cdot 0 \\
29.5\end{array}$ & $\begin{array}{l}22.5 \\
18.5 \\
17.5\end{array}$ & $\begin{array}{l}0.73 \\
5.58 \\
5.57\end{array}$ & $\begin{array}{l}0.10 \\
0.28 \\
0.34\end{array}$ & $\begin{array}{l}57 \cdot 0 \\
22.0 \\
25 \cdot 7\end{array}$ & $\begin{array}{l}7 \cdot 380 \\
7 \cdot 265 \\
7 \cdot 270\end{array}$ & $\begin{array}{l}39 \cdot 0 \\
44 \cdot 0 \\
43 \cdot 0\end{array}$ & $\begin{array}{l}21.5 \\
17.0 \\
16.0\end{array}$ \\
\hline & 30 & $\begin{array}{l}\mathbf{R} \\
\mathbf{E}\end{array}$ & $\begin{array}{l}94.0 \\
95 \cdot 2 \\
94.0\end{array}$ & $\begin{array}{l}7 \cdot 400 \\
7 \cdot 375 \\
7 \cdot 385\end{array}$ & $\begin{array}{l}43 \cdot 0 \\
48 \cdot 0 \\
46 \cdot 0\end{array}$ & $\begin{array}{l}25 \cdot 0 \\
25 \cdot 0 \\
22 \cdot 0\end{array}$ & $\begin{array}{l}0.65 \\
1 \cdot 17 \\
1 \cdot 17\end{array}$ & $\begin{array}{l}0.07 \\
0 \cdot 14 \\
0 \cdot 13\end{array}$ & $\begin{array}{l}66.0 \\
56.0 \\
41.0\end{array}$ & $\begin{array}{l}7 \cdot 365 \\
7 \cdot 345 \\
7 \cdot 345\end{array}$ & $\begin{array}{l}50.0 \\
51.0 \\
51.0\end{array}$ & $\begin{array}{l}25 \cdot 0 \\
23 \cdot 5 \\
23 \cdot 0\end{array}$ \\
\hline & 31 & $\mathbf{R}$ & $97 \cdot 5$ & $7 \cdot 445$ & 31.5 & $23 \cdot 5$ & 1.56 & 0.17 & - & - & - & - \\
\hline & & E & 93.6 & $\stackrel{7 \cdot 420}{-}$ & $\stackrel{33.5}{-}$ & 23.0 & 3.51 & $=$ & 二 & 二 & 三 & 二 \\
\hline & 32 & $\underset{\mathbf{E}}{\mathbf{R}}$ & $\begin{array}{l}94.0 \\
88.6\end{array}$ & $\begin{array}{l}7.430 \\
7.420\end{array}$ & $\begin{array}{l}33 \cdot 5 \\
33.0\end{array}$ & $\begin{array}{l}24.5 \\
22.0\end{array}$ & $\begin{array}{l}0.52 \\
1.63\end{array}$ & 0.08 & $\begin{array}{l}68 \cdot 4 \\
42 \cdot 0\end{array}$ & $\begin{array}{l}7 \cdot 415 \\
7 \cdot 350\end{array}$ & $\begin{array}{l}37.0 \\
41.5 \\
43.0\end{array}$ & $\begin{array}{l}23.0 \\
20.0\end{array}$ \\
\hline & & & $90 \cdot 3$ & $7 \cdot 375$ & $36 \cdot 0$ & $21 \cdot 0$ & $2 \cdot 34$ & $0 \cdot 11$ & $46 \cdot 2$ & $7 \cdot 340$ & 43.0 & $20 \cdot 0$ \\
\hline & 33 & $\underset{\mathbf{E}}{\mathbf{R}}$ & $\begin{array}{l}98.0 \\
89 \cdot 2 \\
88.5\end{array}$ & $\begin{array}{l}7 \cdot 410 \\
7 \cdot 400 \\
7 \cdot 405\end{array}$ & $\begin{array}{l}34.5 \\
36.5 \\
35.0\end{array}$ & $\begin{array}{l}22 \cdot 5 \\
22 \cdot 7 \\
22 \cdot 1\end{array}$ & $\begin{array}{l}0.94 \\
1.65 \\
2.35\end{array}$ & $\bar{z}$ & $\begin{array}{l}72 \cdot 7 \\
45 \cdot 4 \\
49 \cdot 8\end{array}$ & $\begin{array}{l}7 \cdot 390 \\
7 \cdot 375 \\
7 \cdot 360\end{array}$ & $\begin{array}{l}38.0 \\
45.0 \\
46.0\end{array}$ & $\begin{array}{l}22 \cdot 7 \\
24 \cdot 2 \\
23 \cdot 5\end{array}$ \\
\hline & 34 & $\mathbf{R}$ & $97 \cdot 0$ & $7 \cdot 390$ & 40.0 & 23.5 & 0.78 & 0.10 & 77.6 & $7 \cdot 365$ & $44 \cdot 0$ & 22.5 \\
\hline & & $\mathrm{E}$ & $\begin{array}{l}95.6 \\
99.0\end{array}$ & $\begin{array}{l}7 \cdot 380 \\
7 \cdot 370\end{array}$ & $\begin{array}{l}40 \cdot 0 \\
45 \cdot 0\end{array}$ & $\begin{array}{l}23.0 \\
23.5\end{array}$ & $\begin{array}{l}0.80 \\
0.98\end{array}$ & $\begin{array}{l}0.13 \\
0.14\end{array}$ & $\begin{array}{l}59.0 \\
60.3\end{array}$ & $\begin{array}{l}7.335 \\
7.340\end{array}$ & $\begin{array}{l}48.0 \\
51.0\end{array}$ & $\begin{array}{l}21 \cdot 5 \\
22 \cdot 5\end{array}$ \\
\hline & 35 & $\mathbf{R}$ & 95.0 & $7 \cdot 430$ & $33 \cdot 0$ & $22 \cdot 5$ & $1 \cdot 17$ & $0 \cdot 14$ & $67 \cdot 8$ & $7 \cdot 400$ & $39 \cdot 0$ & 23.5 \\
\hline & & & 95.6 & $7 \cdot 420$ & $33 \cdot 0$ & $22 \cdot 0$ & $2 \cdot 21$ & $0 \cdot 19$ & $44 \cdot 4$ & $7 \cdot 370$ & $40 \cdot 0$ & 20.5 \\
\hline & 36 & $\begin{array}{l}\mathbf{R} \\
\mathrm{E}\end{array}$ & $\begin{array}{l}97 \cdot 1 \\
97 \cdot 0 \\
96 \cdot 4\end{array}$ & $\begin{array}{l}7 \cdot 420 \\
7 \cdot 395 \\
7 \cdot 400\end{array}$ & $\begin{array}{l}38 \cdot 0 \\
35 \cdot 5 \\
36 \cdot 5\end{array}$ & $\begin{array}{l}24 \cdot 0 \\
22 \cdot 2 \\
22 \cdot 5\end{array}$ & $\begin{array}{l}0.78 \\
1.43 \\
1.56\end{array}$ & $\begin{array}{l}0.05 \\
0 \cdot 12 \\
0 \cdot 13\end{array}$ & $\begin{array}{l}74 \cdot 2 \\
43.0 \\
56.6\end{array}$ & $\begin{array}{l}7 \cdot 380 \\
7 \cdot 340 \\
7 \cdot 350\end{array}$ & $\begin{array}{l}45.0 \\
47 \cdot 0 \\
47.0\end{array}$ & $\begin{array}{l}23 \cdot 5 \\
21 \cdot 0 \\
21 \cdot 0\end{array}$ \\
\hline & 37 & $\stackrel{\mathrm{R}}{\mathrm{E}}$ & $\begin{array}{l}99 \cdot 0 \\
96 \cdot 3\end{array}$ & $\begin{array}{l}7.400 \\
7.340\end{array}$ & $\begin{array}{r}33.0 \\
36.5\end{array}$ & $\begin{array}{l}21 \cdot 0 \\
20 \cdot 0\end{array}$ & $\begin{array}{l}0 \cdot 78 \\
3 \cdot 25\end{array}$ & $\begin{array}{l}0.08 \\
0.15\end{array}$ & $\begin{array}{l}72 \cdot 0 \\
50 \cdot 3\end{array}$ & $\begin{array}{l}7.395 \\
7.340\end{array}$ & $\begin{array}{l}40.0 \\
49 \cdot 0\end{array}$ & $\begin{array}{l}23.0 \\
21.5\end{array}$ \\
\hline & & & $95 \cdot 4$ & $7 \cdot 320$ & $37 \cdot 0$ & $19 \cdot 0$ & $2 \cdot 60$ & 0.17 & 54.5 & $7 \cdot 315$ & 46.0 & 20.0 \\
\hline & 38 & $\underset{\mathbf{E}}{\mathbf{R}}$ & $\begin{array}{l}95.6 \\
94.7 \\
95.8\end{array}$ & $\begin{array}{l}7 \cdot 365 \\
7 \cdot 385 \\
7 \cdot 360\end{array}$ & $\begin{array}{l}45 \cdot 0 \\
43 \cdot 0 \\
43 \cdot 0\end{array}$ & $\begin{array}{l}23 \cdot 5 \\
24 \cdot 0 \\
23 \cdot 0\end{array}$ & $\begin{array}{l}0.65 \\
1.43 \\
1.56\end{array}$ & $\begin{array}{l}0.05 \\
0.09 \\
0.11\end{array}$ & $\begin{array}{l}72 \cdot 9 \\
46 \cdot 9 \\
46 \cdot 5\end{array}$ & $\begin{array}{l}7 \cdot 345 \\
7 \cdot 345 \\
7 \cdot 310\end{array}$ & $\begin{array}{l}50.0 \\
51.0 \\
45.0\end{array}$ & $\begin{array}{l}23 \cdot 0 \\
22 \cdot 0 \\
22 \cdot 0\end{array}$ \\
\hline & 39 & $\begin{array}{l}\mathbf{R} \\
\mathbf{E}\end{array}$ & $\begin{array}{l}95 \cdot 3 \\
96 \cdot 1 \\
96 \cdot 0\end{array}$ & $\begin{array}{l}7 \cdot 460 \\
7 \cdot 450 \\
7 \cdot 420\end{array}$ & $\begin{array}{l}35.5 \\
36.0 \\
35.5\end{array}$ & $\begin{array}{l}25 \cdot 0 \\
25 \cdot 0 \\
23 \cdot 0\end{array}$ & $\frac{1.30}{1.82}$ & $\frac{0 \cdot 10}{0 \cdot 12}$ & $\begin{array}{l}67 \cdot 8 \\
52 \cdot 2 \\
49 \cdot 0\end{array}$ & $\begin{array}{c}7 \cdot 420 \\
7 \cdot 380 \\
-\end{array}$ & $\begin{array}{r}38.5 \\
48.0 \\
-\end{array}$ & $\begin{array}{r}23.0 \\
24.5 \\
-\end{array}$ \\
\hline
\end{tabular}

Note: During exercise, the observations at the 3rd and 4th minute are shown in the upper line and those in the 9th to 10th minute on the lower.

$\mathrm{SAO}_{2}$, systemic oxygen saturation; $\mathrm{Sṽ}_{2}$, mixed venous oxygen saturation; Bicarb., standard bicarbonate; Lact., lactate; Pyr., pyruvate.

the ventilation and the indirect left atrial pressure on exercise in those patients in whom the latter was measured. A highly significant relationship exists $(\mathrm{p}<0.001)$. The 3 patients amongst these with abnormal arterial gases (i.e. $\mathrm{PCO}_{2}$ more than $45 \mathrm{~mm}$. $\mathrm{Hg}$ and/or systemic arterial oxygen saturation less than $90 \%$ ) are shown in solid symbols. It is seen that their ventilatory response falls into line with the rest and does not suggest that an additional humoral factor is operating.

The changes in the venous blood chemistry merit consideration. Since, with few exceptions, the arterial oxygen saturation showed no significant variations, the venous oxygen saturation for a given exercise load was a direct function of the cardiac output. No significant relationship between the latter and the ventilation was found during mild supine exercise in a much larger series of patients previously reported (Gazetopoulos et al., 1966), and it is, therefore, unlikely that the venous oxygen saturation is related directly to the ventilatory response under those conditions. 


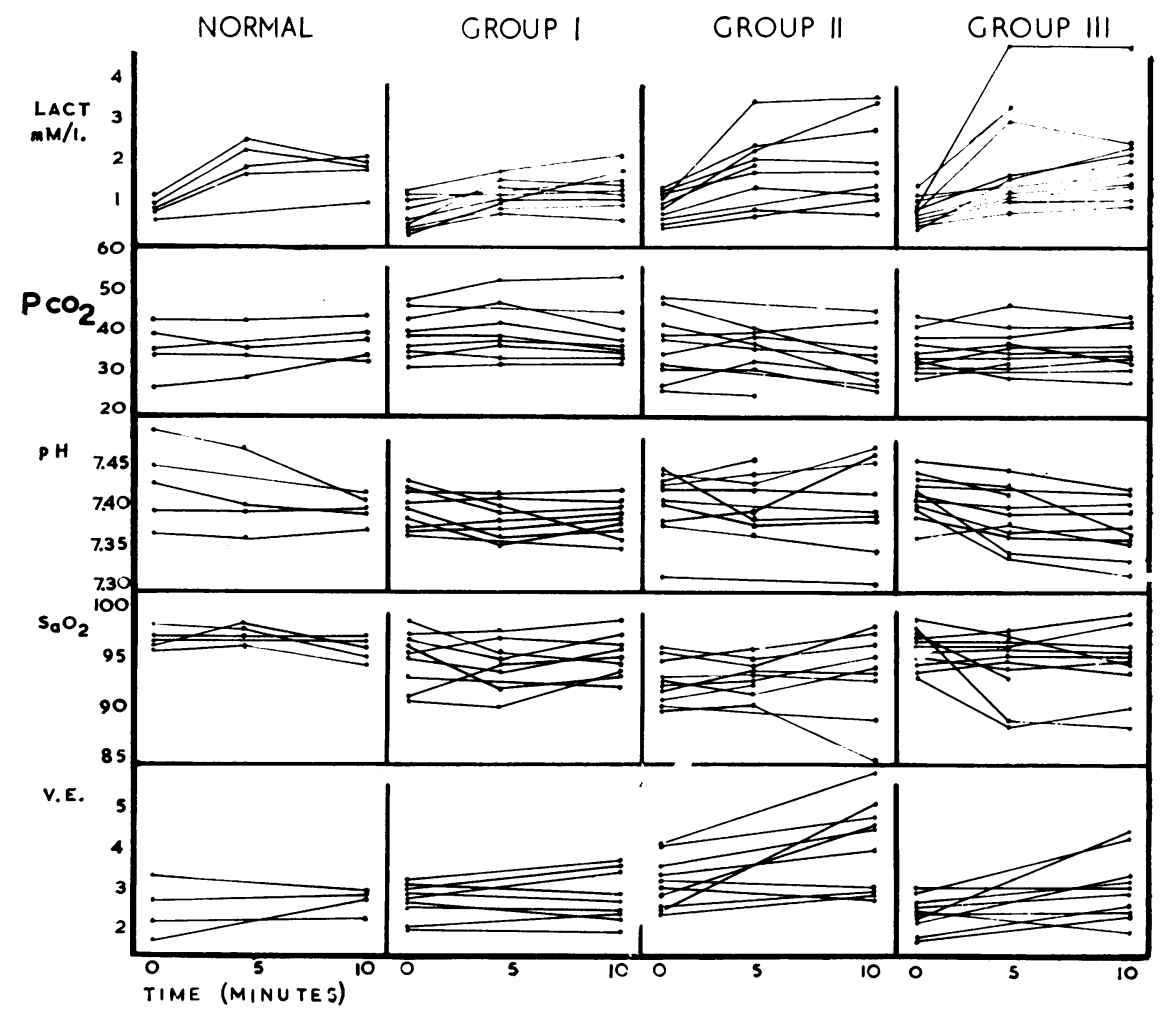

Fig. 1.-Ventilation (expressed as ventilatory equivalent, VE) in relation to arterial oxygen saturation, $p H$, $\mathrm{PCO}_{2}$, and lactate concentration at rest and during supine exercise in normal subjects, and several types of heart disease. Group I, left ventricular disease; Group II, mitral stenosis; Group III, pulmonary stenosis. For blood chemistry the three points shown represent, respectively, rest, 3rd to 4th minute, and 8th to 10th minutes of exercise. For ventilation, resting, and steady-state, 5th to 10 th minute values are given.

Likewise the mixed venous $\mathrm{PCO}_{2}$ depends not only on the arteriovenous difference but also on the level of the arterial $\mathrm{PCO}_{2}$; and it is evident from Fig. 2 that there was no significant difference in the former between the several groups. The venous $\mathrm{PCO}_{2}$ is plotted against the ventilation in Fig. 7 . The solid line in this figure shows a postulated normal ventilatory response to increasing venous $\mathrm{PCO}_{2}$ given by Riley et al. (1963). It is clear from our data that patients with the higher ventilation in all groups tended to have lower rather than higher levels of venous $\mathrm{PCO}_{2}$. Only a few of our patients have a significantly raised venous $\mathrm{PCO}_{2}$ during exercise and they include those with chronic $\mathrm{CO}_{2}$ retention; the ventilation in these patients is in the lower range.

In the upper part of Fig. 2 is shown the mixed venous $p H$. When this is plotted against the ventilatory response to exercise (Fig. 8) it is seen that the patients with the greatest ventilation were not those who showed the lowest venous $p \mathrm{H}$. From
Fig. 7 and 8 it is also seen that there was no difference in the mixed venous $p \mathrm{H}$ and $\mathrm{PCO}_{2}$ between patients with normal (open symbols) and low cardiac output (closed symbols), in spite of the wider arteriovenous difference in the low-output patients.

B. Upright Exercise. Table IV gives the changes seen in the patients studied on the bicycle ergometer.

Figure 9 illustrates the 4 patients with mitral stenosis. Of these, 3 were unable to complete more than a few minutes of exercise at the second level owing to dyspnœa. Accordingly no measurements of the ventilation at that stage could be made in them, though it was clear that considerable hyperventilation was present, certainly more than $40 \mathrm{l} . / \mathrm{min}$. The ventilation was also excessive at the first level in these 3 patients. In all the $\mathrm{PCO}_{2}$ was low, the $p \mathrm{H}$ high, and there was no abnormal rise in lactate nor fall in arterial saturation. The fourth patient, who had moderate mitral stenosis, 


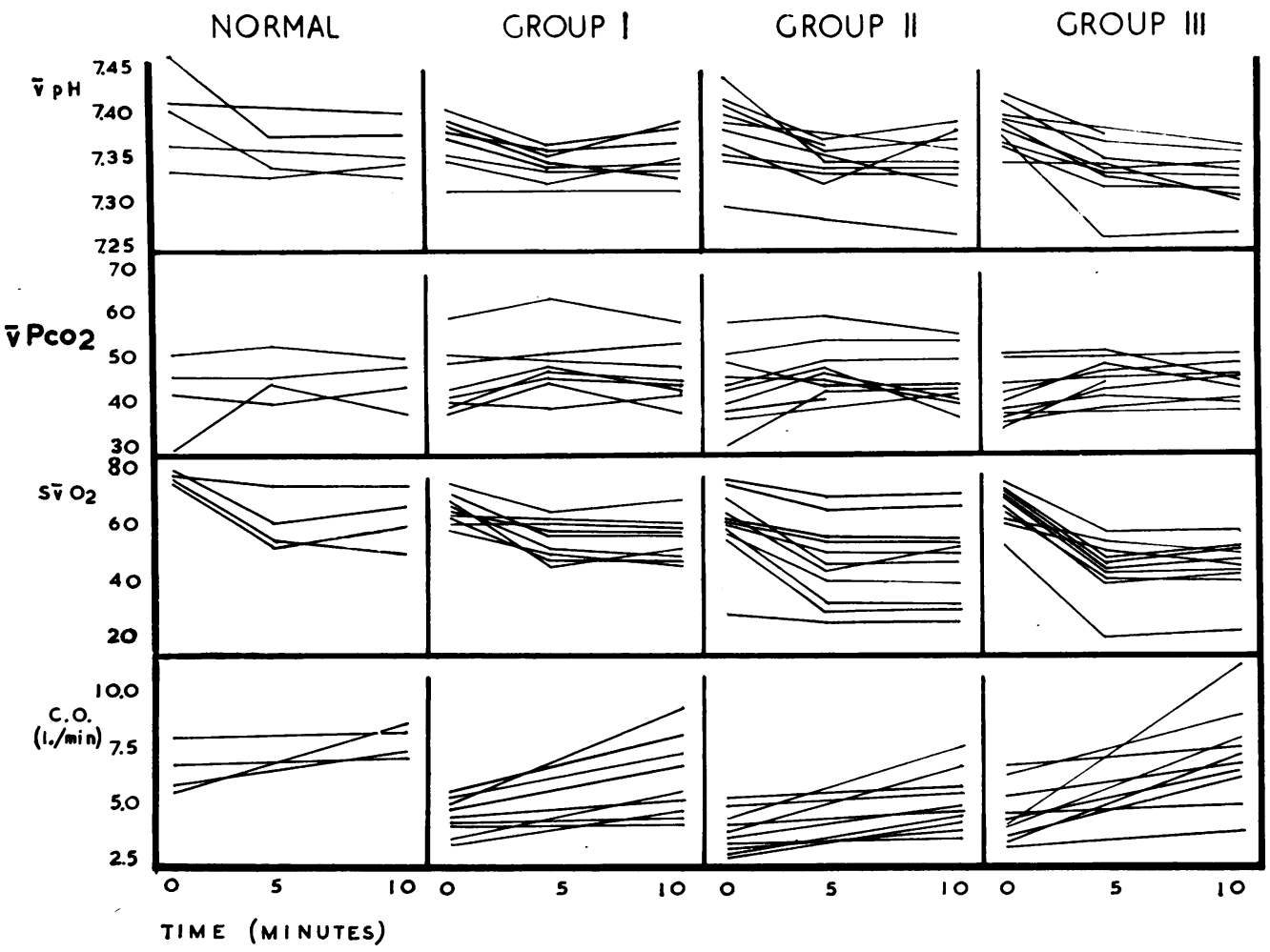

FIg. 2.-Cardiac output in relation to mixed venous chemistry. Definitions as in Fig. 1. Observations were made simultaneously with those of Fig. 1.

performed the first exercise level with a normal ventilatory response. The mean pulmonary arterial wedge pressure had been $29 \mathrm{~mm}$. $\mathrm{Hg}$ at an exercise level corresponding to an oxygen uptake of 530 $\mathrm{ml} . / \mathrm{min}$. in the supine position during cardiac catheterization. It is likely that at the first level of exercise when the posture was upright and the oxygen uptake lower $(445 \mathrm{ml} . / \mathrm{min}$.) the wedge pressure was also lower. Hyperventilation was present when the exercise load was increased and the lactate rose

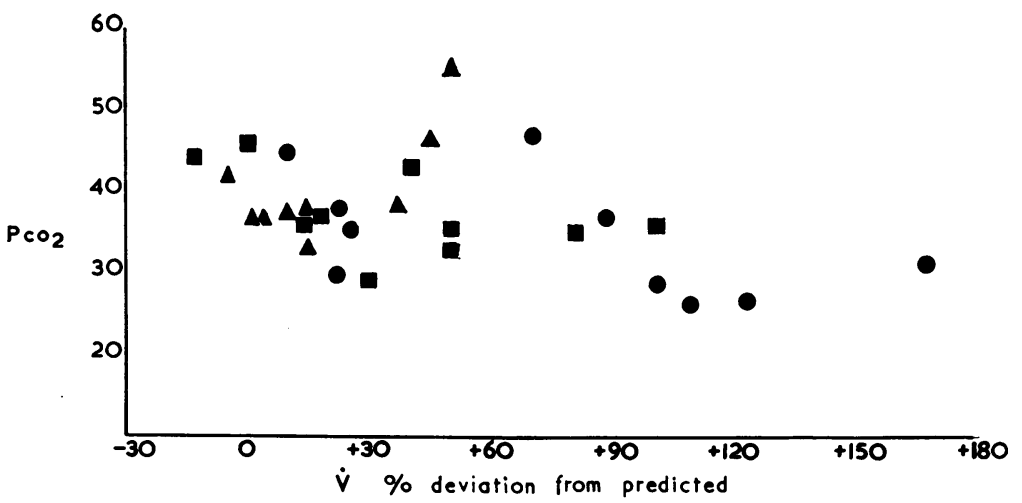

FIG. 3.-Arterial $\mathrm{PcO}_{2}$ and ventilatory response to exercise in all groups expressed as deviations from mean predicted values (Gazetopoulos et al., 1966). Triangles represent Group I, circles Group II, squares Group III. The $\mathrm{PCO}_{2}$ is seen broadly to vary inversely with the degree of hyperventilation. 


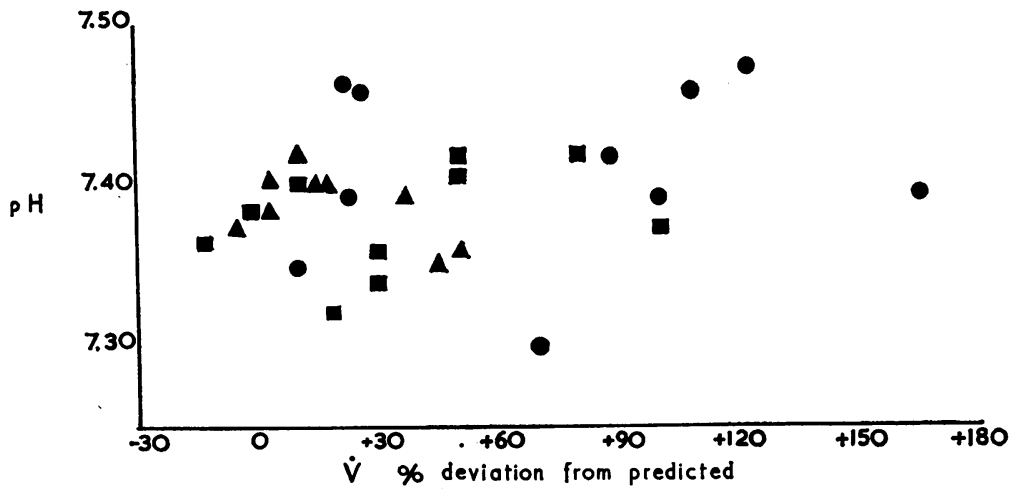

FIg. 4.-Arterial $p \mathrm{H}$ and ventilation. Symbols as in Fig. 3. Patients with greater ventilation are the more alkalotic.

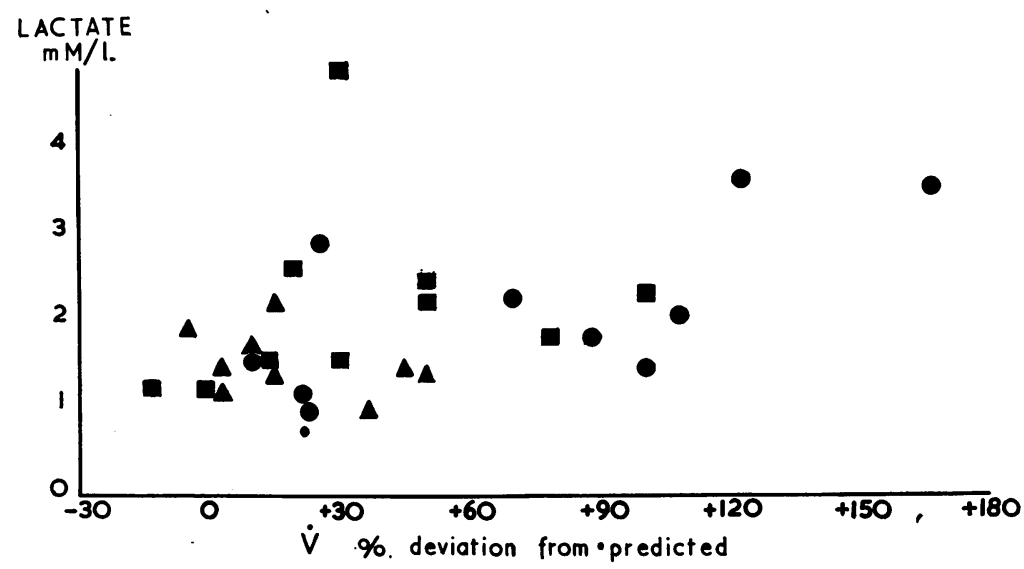

Fig. 5.-Arterial lactate concentration and ventilation. Symbols as in Fig. 3. No significant relationship is present $(\mathrm{r}=0 \cdot 12 ; \mathrm{p}>0 \cdot 1)$.

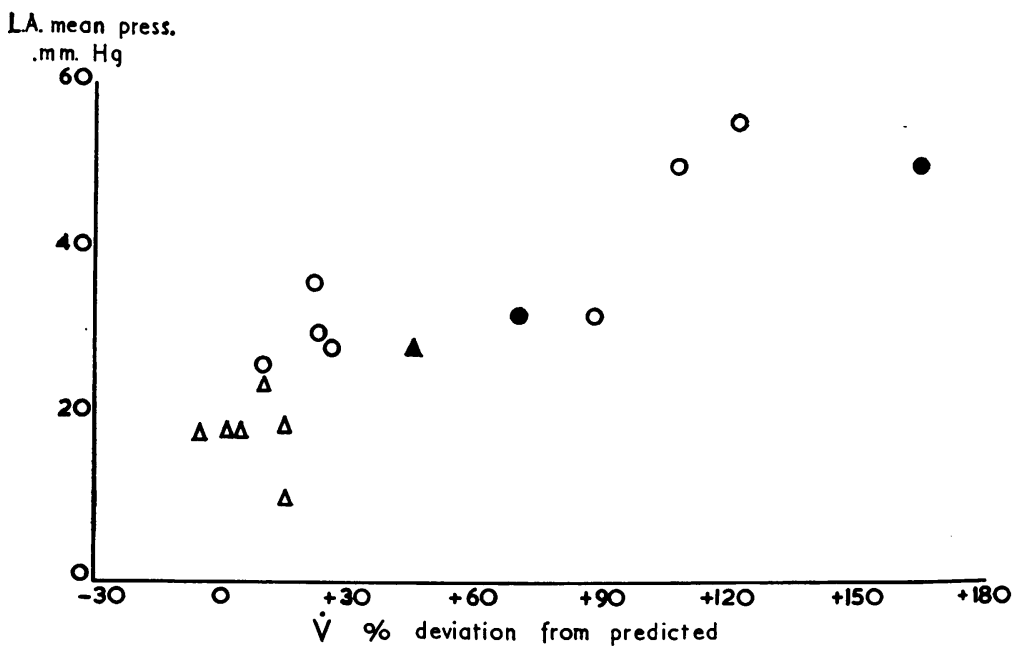

FIG. 6.-Ventilation and left atrial mean pressure on exercise in patients where blood gas measurements were made. The closed symbols represent those patients with additional hypercapnia or hypoxæmia or both. Despite the abnormal blood chemistry the ventilatory response is not remarkable, suggesting that this does not represent an additional stimulus. Symbols as in Fig. 3. A significant relationship exists between the two variables $(r=0.987 ; p<0.001)$. 


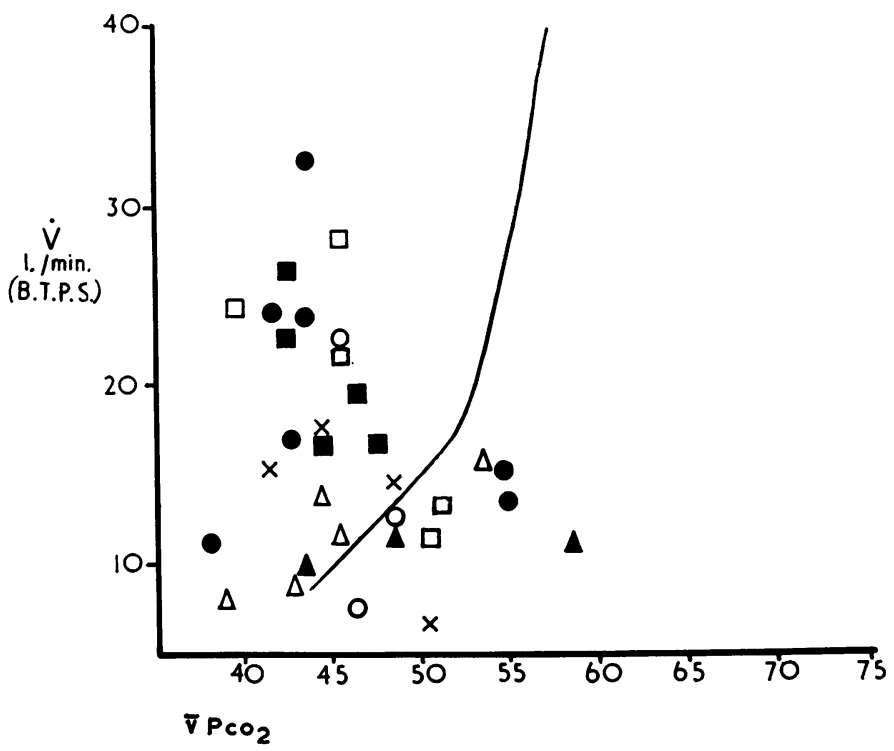

Fig. 7.-Mixed venous. $\mathrm{PcO}_{2}$ and ventilation. Crosses represent normal subjects, otherwise symbols as in Fig. 3. Open symbols signify normal cardiac output on exercise; closed symbols impaired output (Gazetopoulos et al., 1966). The solid line is the ventilation-venous $\mathrm{PCO}_{2}$ relationship given by Riley et al. (1963). Higher ventilation is associated with lower rather than higher venous $\mathbf{P C O}_{2}$.

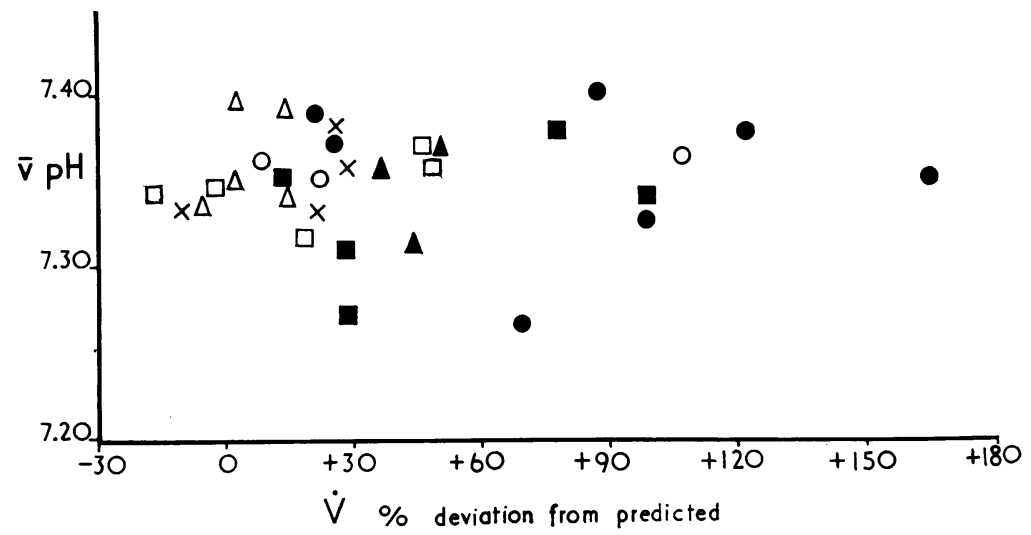

FIG. 8.-Mixed venous $p H$ and ventilation. Symbols as in Fig. 7. Higher ventilatory levels are not associated with a tendency towards venous acidæmia.

above normal. The arterial $\mathrm{PCO}_{2}$ at this stage was falling and there was only slight tendency towards acidæmia.

Figure 10 shows our findings in patients with pulmonary stenosis studied on the bicycle ergometer. The lowermost part of this figure shows the ventilation, expressed as minute volume, and the heart rate, the limits of normality being those derived from published data and from our own findings (Gazetopoulos et al., 1966). Above that are illustrated the changes in arterial oxygen saturation, $\mathrm{PCO}_{2}, \mathrm{pH}$, and lactate. All patients were exercised to their maximal working capacity, at which point they had to stop on account of dyspncea or fatigue. The ventilatory response was usually excessive at all points up to the highest level. Lactate production was somewhat excessive (see below). We noted a reduction in exercise tolerance in all these patients that seemed to be related to the severity of the stenosis and to their age. In all cases the $p \mathrm{H}$ and $\mathrm{PcO}_{2}$ 
TABLE IV

ARTERIAL BLOOD COMPOSITION AND OTHER DATA AT REST AND DURING GRADUATED INCREASES OF UPRIGHT EXERCISE ON BICYCLE ERGOMETER

\begin{tabular}{|c|c|c|c|c|c|c|c|c|c|c|c|c|}
\hline \multirow{2}{*}{\multicolumn{2}{|c|}{$\begin{array}{l}\text { Case No. and } \\
\text { diagn. }\end{array}$}} & & \multirow{2}{*}{$\begin{array}{c}\text { Ex. load } \\
(\mathrm{kg} . / \mathrm{min} .)\end{array}$} & \multirow{2}{*}{ 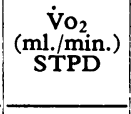 } & \multirow{2}{*}{$\begin{array}{l}\text { Heart } \\
\text { rate }\end{array}$} & \multirow{2}{*}{$\underset{\text { BTPS }}{\mathrm{V}}$} & \multirow{2}{*}{\multicolumn{6}{|c|}{ Arterial blood }} \\
\hline & & & & & & & & & & & & \\
\hline \multirow[t]{2}{*}{25} & MS & $\begin{array}{l}\mathbf{R} \\
\mathbf{E}\end{array}$ & $\begin{array}{r}0 \\
200 \\
400\end{array}$ & $\begin{array}{r}200 \\
445 \\
1205\end{array}$ & $\begin{array}{r}64 \\
88 \\
120\end{array}$ & $\begin{array}{r}6 \cdot 3 \\
15 \cdot 6 \\
32 \cdot 4\end{array}$ & $\begin{array}{c}96.0 \\
= \\
\overline{98.0}\end{array}$ & $\begin{array}{l}7 \cdot 405 \\
7 \cdot 405 \\
7 \cdot 415 \\
7 \cdot 415 \\
7 \cdot 390\end{array}$ & $\begin{array}{l}45 \cdot 0 \\
44 \cdot 0 \\
40 \cdot 0 \\
37 \cdot 0 \\
34 \cdot 0\end{array}$ & $\begin{array}{l}26 \cdot 0 \\
25 \cdot 0 \\
24 \cdot 5 \\
23 \cdot 0 \\
22.5\end{array}$ & $\begin{array}{l}1.49 \\
2.05 \\
2 \cdot 14 \\
5.07 \\
5.46\end{array}$ & $\begin{array}{l}0.08 \\
0.10 \\
0.11 \\
0.12 \\
0.20\end{array}$ \\
\hline & & Rec. $\begin{array}{l}\left(5^{\prime}\right) \\
\left(10^{\prime}\right)\end{array}$ & & - & $\begin{array}{l}72 \\
72\end{array}$ & $\begin{array}{l}8 \cdot 6 \\
6 \cdot 1\end{array}$ & 二 & $\begin{array}{l}7 \cdot 365 \\
7 \cdot 380\end{array}$ & $\begin{array}{l}42 \cdot 0 \\
40 \cdot 0\end{array}$ & $\begin{array}{l}23 \cdot 0 \\
23 \cdot 0\end{array}$ & $\begin{array}{l}4 \cdot 29 \\
3 \cdot 08\end{array}$ & $\begin{array}{l}0.35 \\
0 \cdot 16\end{array}$ \\
\hline & MS & $\begin{array}{l}\mathbf{R} \\
\mathbf{E}\end{array}$ & $\begin{array}{r}0 \\
200 \\
400\end{array}$ & $\begin{array}{l}190 \\
912 \\
=\end{array}$ & $\begin{array}{r}84 \\
\frac{132}{160}\end{array}$ & $\begin{array}{r}9 \cdot 5 \\
28 \cdot 0 \\
-\end{array}$ & $\begin{array}{l}97 \cdot 0 \\
\overline{97 \cdot 2} \\
97 \cdot 0\end{array}$ & $\begin{array}{l}7 \cdot 535 \\
7 \cdot 505 \\
7 \cdot 505 \\
7 \cdot 500\end{array}$ & $\begin{array}{l}32.5 \\
30.5 \\
30.5 \\
30.5\end{array}$ & $\begin{array}{l}27 \cdot 0 \\
24 \cdot 5 \\
24 \cdot 5 \\
24 \cdot 0\end{array}$ & $\begin{array}{l}1 \cdot 10 \\
2 \cdot 30 \\
2 \cdot 10 \\
2 \cdot 20\end{array}$ & $\begin{array}{l}0.08 \\
0.11 \\
0.13 \\
0.16\end{array}$ \\
\hline & & Rec. $\left(5^{\prime}\right)$ & - & - & - & - & $\overline{-}$ & $\overline{7 \cdot 485}$ & $\overline{32 \cdot 0}$ & $\overline{24 \cdot 5}$ & $\overline{1 \cdot 80}$ & $\overline{0.12}$ \\
\hline \multirow[t]{2}{*}{27} & MS & $\begin{array}{l}\mathbf{R} \\
\mathbf{E}\end{array}$ & $\begin{array}{r}0 \\
200 \\
400\end{array}$ & $\begin{array}{l}298 \\
920 \\
-\end{array}$ & $\begin{array}{r}80 \\
128 \\
136\end{array}$ & $\begin{array}{r}11 \cdot 0 \\
36 \cdot 0 \\
-\end{array}$ & $\frac{97 \cdot 0}{\overline{97 \cdot 0}}$ & $\begin{array}{l}7 \cdot 470 \\
7 \cdot 460 \\
7 \cdot 460 \\
7 \cdot 440\end{array}$ & $\begin{array}{l}32.0 \\
30.0 \\
28.0 \\
29.0\end{array}$ & $\begin{array}{l}24 \cdot 0 \\
22 \cdot 0 \\
21 \cdot 0 \\
21 \cdot 0\end{array}$ & $\begin{array}{l}0.65 \\
1.36 \\
1.95 \\
2.01\end{array}$ & $\begin{array}{l}0.09 \\
0.12 \\
0.16 \\
0.16\end{array}$ \\
\hline & & Rec. $\left(10^{\prime}\right)$ & & - & 80 & $12 \cdot 0$ & 二 & $\overline{7 \cdot 450}$ & $\overline{30 \cdot 0}$ & $\overline{22 \cdot 0}$ & $\overline{1.49}$ & $\overline{0 \cdot 18}$ \\
\hline \multirow[t]{2}{*}{28} & MS & $\underset{\mathbf{E}}{\mathbf{R}}$ & $\begin{array}{r}0 \\
200 \\
400\end{array}$ & $\begin{array}{l}200 \\
604 \\
-\end{array}$ & $\begin{array}{l}100 \\
140 \\
160\end{array}$ & $\begin{array}{r}9 \cdot 0 \\
33.0 \\
-\end{array}$ & $\frac{95 \cdot 0}{95.6}$ & $\begin{array}{l}7 \cdot 415 \\
7 \cdot 450 \\
7 \cdot 460 \\
7 \cdot 470\end{array}$ & $\begin{array}{l}41 \cdot 0 \\
29.5 \\
25 \cdot 0 \\
24.5\end{array}$ & $\begin{array}{l}25.0 \\
22.5 \\
21.0 \\
21.5\end{array}$ & $\begin{array}{l}1 \cdot 23 \\
2 \cdot 34 \\
2 \cdot 73 \\
3 \cdot 12\end{array}$ & $\begin{array}{l}0.07 \\
0.12 \\
0.13 \\
0.13\end{array}$ \\
\hline & & Rec. $\begin{array}{l}\left(5^{\prime}\right) \\
\left(10^{\prime}\right)\end{array}$ & & - & $\begin{array}{l}125 \\
120\end{array}$ & - & E & $\overline{7} \overline{7 \cdot 500}$ & $\overline{24 \cdot 0}$ & $\overline{23 \cdot 0}$ & $\begin{array}{l}2 \overline{1.14} \\
1.95\end{array}$ & $\begin{array}{l}0 \cdot \overline{13} \\
0 \cdot 11\end{array}$ \\
\hline \multirow[t]{3}{*}{35} & PS & $\underset{\mathbf{E}}{\mathbf{R}}$ & $\begin{array}{r}0 \\
200\end{array}$ & $\begin{array}{l}350 \\
800\end{array}$ & $\begin{array}{l}80 \\
88\end{array}$ & $\begin{array}{l}10 \cdot 0 \\
18 \cdot 0\end{array}$ & $98 \cdot 0$ & $7 \cdot 440$ & 33.0 & $\underline{22 \cdot 5}$ & 1.49 & $\underline{0.12}$ \\
\hline & & & $\begin{array}{l}400 \\
600 \\
800\end{array}$ & $\begin{array}{c}1030 \\
1610 \\
-\end{array}$ & $\begin{array}{l}100 \\
132 \\
170\end{array}$ & $\begin{array}{l}20.0 \\
31.5 \\
45.0\end{array}$ & $\begin{array}{l}98 \cdot 0 \\
98 \cdot 0 \\
98 \cdot 0 \\
96 \cdot 0 \\
96 \cdot 2 \\
92 \cdot 3\end{array}$ & $\begin{array}{c}7 \cdot 470 \\
7 \cdot 440 \\
7 \cdot 460 \\
7 \cdot 425 \\
7 \cdot 400 \\
7 \cdot 360\end{array}$ & $\begin{array}{l}30.5 \\
32.0 \\
33.0 \\
33.0 \\
32.0 \\
29.5 \\
-\end{array}$ & $\begin{array}{l}22.5 \\
22 \cdot 0 \\
23.5 \\
22 \cdot 0 \\
20.5 \\
17.5\end{array}$ & $\begin{array}{l}1.62 \\
1.82 \\
1.75 \\
3.05 \\
4.03 \\
8.45\end{array}$ & $\begin{array}{l}0.13 \\
0.20 \\
0.19 \\
0.18 \\
0.19 \\
0.21\end{array}$ \\
\hline & & Rec. $\left(10^{\prime}\right)$ & & - & - & - & $9 \overline{98.0}$ & $\overline{7 \cdot 430}$ & $2 \overline{27 \cdot 0}$ & $\overline{19 \cdot 5}$ & $5 \cdot \overline{85}$ & $0 \cdot \overline{31}$ \\
\hline & PS & $\begin{array}{l}\mathbf{R} \\
\mathbf{E}\end{array}$ & $\begin{array}{r}0 \\
200 \\
400 \\
600\end{array}$ & $\begin{array}{r}250 \\
865 \\
1225 \\
1600\end{array}$ & $\begin{array}{r}64 \\
100 \\
120 \\
162\end{array}$ & $\begin{array}{r}7 \cdot 8 \\
21 \cdot 0 \\
30 \cdot 7 \\
40 \cdot 0\end{array}$ & $\begin{array}{l}97 \cdot 8 \\
97 \cdot 6 \\
96 \cdot 1 \\
97 \cdot 3 \\
96 \cdot 7 \\
95 \cdot 3\end{array}$ & $\begin{array}{l}7 \cdot 420 \\
7 \cdot 480 \\
7 \cdot 450 \\
7 \cdot 460 \\
7 \cdot 425 \\
7 \cdot 415\end{array}$ & $\begin{array}{l}37.0 \\
25 \cdot 5 \\
28 \cdot 0 \\
27 \cdot 5 \\
29 \cdot 0 \\
30.0\end{array}$ & $\begin{array}{l}24 \cdot 0 \\
22 \cdot 5 \\
22 \cdot 0 \\
22 \cdot 5 \\
21 \cdot 5 \\
21 \cdot 3\end{array}$ & $\begin{array}{l}0.98 \\
2.01 \\
2.34 \\
2.92 \\
2.93 \\
3.30\end{array}$ & $\begin{array}{l}0.06 \\
0.16 \\
0.18 \\
0.18 \\
0.19 \\
0.20\end{array}$ \\
\hline & & & 800 & - & 180 & $45 \cdot 8$ & 二 & 二 & E & 二 & E & $\bar{z}$ \\
\hline 37 & PS & $\begin{array}{l}\mathbf{R} \\
\mathbf{E} \\
\text { Rec. } \stackrel{\left(5^{\prime}\right)}{\left(15^{\prime}\right)}\end{array}$ & $\begin{array}{r}0 \\
200 \\
400 \\
600\end{array}$ & $\begin{array}{r}270 \\
810 \\
1080 \\
- \\
- \\
-\end{array}$ & $\begin{array}{r}76 \\
108 \\
140 \\
182 \\
100 \\
95\end{array}$ & $\begin{array}{r}8 \cdot 5 \\
19 \cdot 0 \\
29 \cdot 5 \\
37 \cdot 4 \\
10 \cdot 8 \\
12 \cdot 0\end{array}$ & $\begin{array}{l}95 \cdot 6 \\
96 \cdot 9 \\
96 \cdot 6 \\
94 \cdot 4 \\
95 \cdot 4 \\
95 \cdot 0 \\
94 \cdot 0 \\
95 \cdot 4 \\
92 \cdot 0\end{array}$ & $\begin{array}{l}\mathbf{7} \cdot 445 \\
\mathbf{7} \cdot 435 \\
\mathbf{7} \cdot 435 \\
\mathbf{7} \cdot 420 \\
\mathbf{7} \cdot 350 \\
\mathbf{7} \cdot 395 \\
\mathbf{7} \cdot 355 \\
\mathbf{7} \cdot 395 \\
\mathbf{7} \cdot 420\end{array}$ & $\begin{array}{l}38 \cdot 0 \\
30 \cdot 0 \\
30 \cdot 5 \\
29 \cdot 0 \\
40 \cdot 0 \\
32 \cdot 0 \\
37 \cdot 5 \\
30 \cdot 0 \\
30 \cdot 0\end{array}$ & $\begin{array}{l}25 \cdot 5 \\
22.5 \\
23 \cdot 0 \\
21 \cdot 0 \\
21 \cdot 0 \\
21 \cdot 0 \\
20 \cdot 5 \\
20.5 \\
21.5\end{array}$ & $\begin{array}{l}1 \cdot 04 \\
1.49 \\
1.56 \\
2 \cdot 47 \\
3.90 \\
4.55 \\
4.65 \\
5 \cdot 46 \\
2 \cdot 86\end{array}$ & $\begin{array}{l}0.06 \\
0.12 \\
0.13 \\
0.17 \\
0.17 \\
0.18 \\
0.18 \\
0.32 \\
0.24\end{array}$ \\
\hline \multirow[t]{2}{*}{38} & PS & $\begin{array}{l}\mathbf{R} \\
\mathbf{E}\end{array}$ & $\begin{array}{r}0 \\
200 \\
400 \\
600\end{array}$ & $\begin{array}{r}255 \\
625 \\
1125 \\
-\end{array}$ & $\begin{array}{r}98 \\
140 \\
164 \\
180\end{array}$ & $\begin{array}{l}11 \cdot 0 \\
21 \cdot 6 \\
30 \cdot 7 \\
43 \cdot 2\end{array}$ & $\begin{array}{l}94 \cdot 7 \\
98.6 \\
95 \cdot 7 \\
96.0 \\
97 \cdot 0 \\
95.7\end{array}$ & $\begin{array}{l}\mathbf{7} \cdot 460 \\
7 \cdot 400 \\
7 \cdot 425 \\
\mathbf{7} \cdot 420 \\
\mathbf{7} \cdot 400 \\
\mathbf{7} \cdot 415\end{array}$ & $\begin{array}{l}31 \cdot 0 \\
35 \cdot 5 \\
35 \cdot 0 \\
34 \cdot 0 \\
34 \cdot 0 \\
31 \cdot 0\end{array}$ & $\begin{array}{l}23.5 \\
21.5 \\
23.5 \\
23.0 \\
22.0 \\
21.5\end{array}$ & $\begin{array}{l}1.04 \\
1.50 \\
1.75 \\
2.53 \\
3.64 \\
46.8\end{array}$ & $\begin{array}{l}0.08 \\
0.10 \\
0.11 \\
= \\
=\end{array}$ \\
\hline & & Rec. $\left(10^{\prime}\right)$ & & - & - & - & $\overline{96.0}$ & $\overline{7 \cdot 425}$ & $\overline{31 \cdot 5}$ & $\overline{22 \cdot 0}$ & $\overline{3 \cdot 60}$ & $\overline{0 \cdot 20}$ \\
\hline 39 & PS & $\begin{array}{l}\mathbf{R} \\
\mathbf{E} \\
\text { Rec. } \stackrel{\left(5^{\prime}\right)}{\left(10^{\prime}\right)}\end{array}$ & $\begin{array}{r}0 \\
200 \\
400\end{array}$ & $\begin{array}{r}408 \\
816 \\
1120 \\
- \\
-\end{array}$ & $\begin{array}{r}68 \\
98 \\
176 \\
80 \\
64\end{array}$ & $\begin{array}{l}11 \cdot 9 \\
27 \cdot 2 \\
45 \cdot 7 \\
11 \cdot 7 \\
10 \cdot 1\end{array}$ & $\begin{array}{c}96 \cdot 0 \\
\bar{Z} \\
\overline{96 \cdot 2} \\
=\end{array}$ & $\begin{array}{l}7 \cdot 495 \\
7 \cdot 440 \\
7 \cdot 450 \\
7 \cdot 455 \\
7 \cdot 460 \\
7 \cdot 415 \\
7 \cdot 450\end{array}$ & $\begin{array}{l}32 \cdot 0 \\
34 \cdot 0 \\
32 \cdot 5 \\
32 \cdot 0 \\
31 \cdot 0 \\
34 \cdot 0 \\
32 \cdot 0\end{array}$ & $\begin{array}{l}25 \cdot 0 \\
23 \cdot 5 \\
22 \cdot 5 \\
22 \cdot 5 \\
22.5 \\
22 \cdot 0 \\
22 \cdot 5\end{array}$ & $\begin{array}{l}0.84 \\
2.99 \\
2.73 \\
3.51 \\
3.90 \\
3.64 \\
2.21\end{array}$ & $\begin{array}{l}0.07 \\
0.12 \\
0.12 \\
0.14 \\
0.16 \\
0.14\end{array}$ \\
\hline 40 & PS & $\begin{array}{l}\mathbf{R} \\
\mathbf{E} \\
\mathbf{R e c} \cdot\left(10^{\prime}\right)\end{array}$ & $\begin{array}{r}0 \\
200\end{array}$ & $\begin{array}{l}290 \\
700 \\
-\end{array}$ & $\begin{array}{l}108 \\
176 \\
108\end{array}$ & $\begin{array}{r}8 \cdot 5 \\
20 \cdot 5 \\
8 \cdot 3\end{array}$ & $\begin{array}{l}96.0 \\
90.0 \\
95.0 \\
93.0\end{array}$ & $\begin{array}{l}7 \cdot 445 \\
7 \cdot 435 \\
7 \cdot 430 \\
7 \cdot 440\end{array}$ & $\begin{array}{l}36 \cdot 0 \\
27 \cdot 0 \\
31 \cdot 0 \\
31 \cdot 5\end{array}$ & $\begin{array}{l}25 \cdot 0 \\
21 \cdot 2 \\
22 \cdot 5 \\
23 \cdot 4\end{array}$ & $\begin{array}{l}1.82 \\
3 \cdot 12 \\
3.64 \\
2.86\end{array}$ & $\begin{array}{l}0.11 \\
0.22 \\
0.19 \\
0.14\end{array}$ \\
\hline
\end{tabular}

Note: All patients were exercised to their maximum ability. Recovery figures are also given. Symbols as in Tables II and III. 


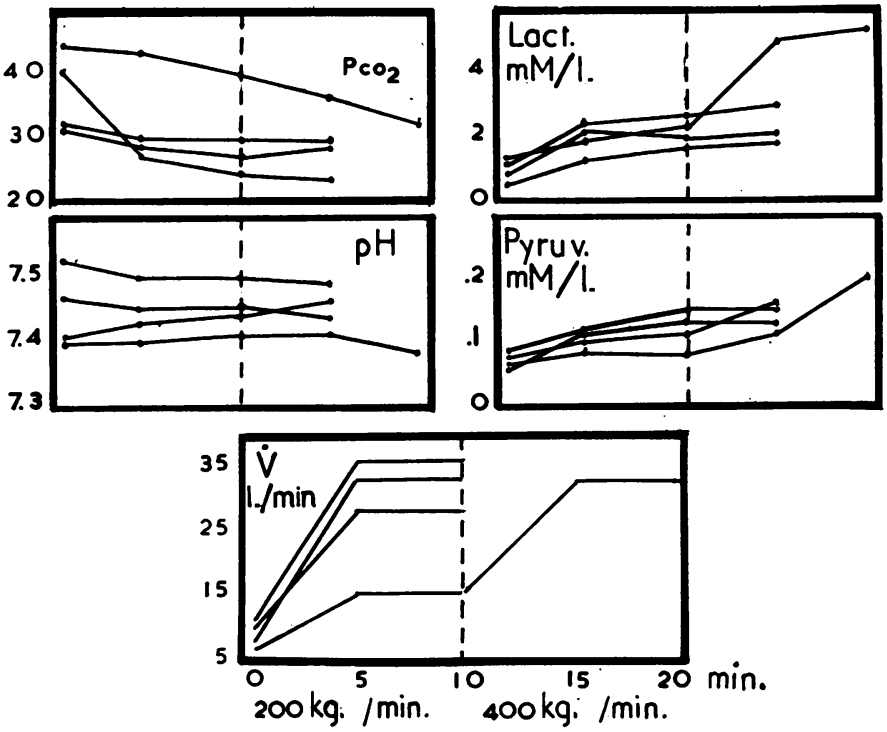

FIG. 9.-Ventilation, arterial $\mathrm{pH}, \mathrm{PCO}_{2}$, lactate, and pyruvate in 4 patients with mitral stenosis at two exercise levels performed upright on the bicycle ergometer. In each case abscissa as in lower part of figure. Note that 3 of the patients were unable to complete 10 minutes of exercise at the higher level. See text for discussion.

showed changes that could have been consequent upon hyperventilation, and the oxygen saturation did not change significantly.

\section{Discussion}

The object of these studies was to observe whether there were any alterations in the blood chemistry that were uniformly associated with hyperventilation or dyspnœa.

The results indicate that in all the groups of patients studied, the variations in blood chemistry were those that would be anticipated as a result of hyperventilation rather than as a cause of it.

In the patients with pulmonary venous hypertension, the $\mathrm{PCO}_{2}$ usually fell and the $p \mathrm{H}$ remained

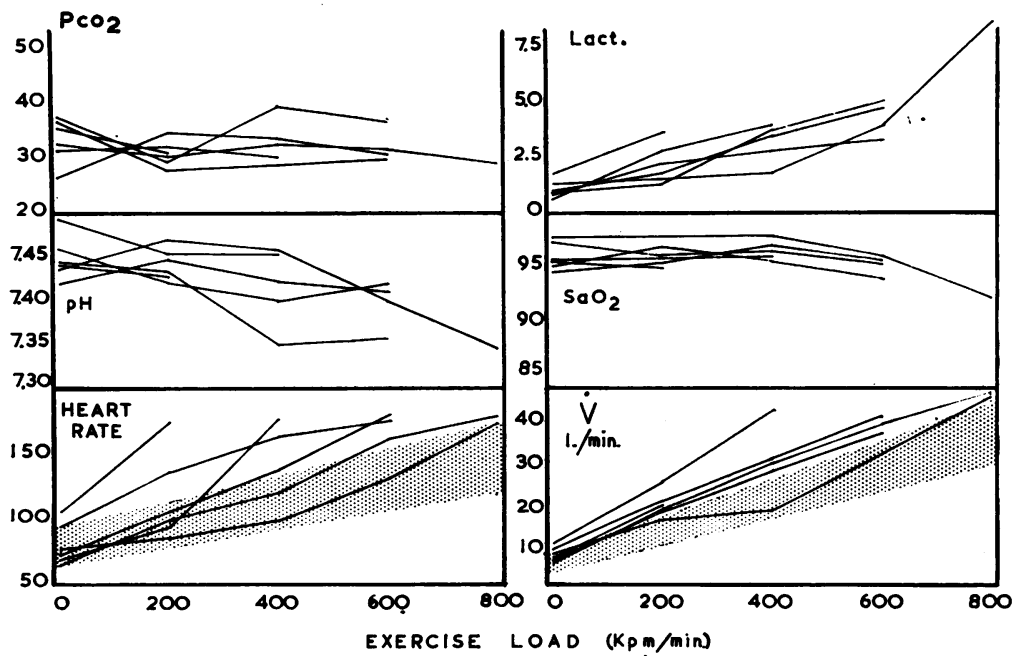

FIG. 10.-Ventilation, heart rate, and blood chemistry in patients with pulmonary stenosis exercised in upright position by graduated increase to maximum working capacity. The normal limits of heart rate and ventilation are shown (Davies et al., 1965). Excessive ventilatory response is usually seen and does not appear to be consequent on changes in blood chemistry. 
in the alkalotic-to-normal range, while the lactate, though sometimes higher than is usually seen in normal subjects, was not uniformly excessive when hyperventilation was present. This observation applies to both the arterial and venous sides of the circulation and supports the view that here the dominant stimulus to hyperventilation is the pulmonary congestion, and that the blood chemical changes reflect the effects of the overbreathing. It is true that some exceptions to this general pattern were seen and a few patients showed hypercapnia or arterial hypoxæmia or both, at rest and on exercise. These patients, however, usually had additional lung disease, and their ventilatory response was similar to those in the rest of the group who had equally high left atrial pressures (Fig. 6). This suggests that additional mild hypercapnia or hypoxæmia has little or nothing to do with stimulating ventilation, when the left atrial pressure is raised. This is not surprising since the adaptation occurring in cases with chronic disturbances of blood chemistry is well recognized; the accommodation to chronic hypoxæmia has been discussed in another study of cyanotic patients (Davies and Gazetopoulos, 1965), where it was often found that there was a normal ventilatory response to exercise in spite of marked hypoxæmia. The adaptation to chronic hypercapnia has also been known for many years (Scott, 1920; Donald and Christie, 1949; Fishman, Turino, and Bergofsky, 1957). We note, however, that hypoxæmia and hypercapnia are seen only exceptionally and we believe that the finding of an increase in arterial $\mathrm{PCO}_{2}$ in a great proportion of the patients of Mauck et al. (1964) was due to early sampling (within the first minute of exercise). Such early increases in $\mathrm{PcO}_{2}$ we have observed both in normal subjects and in patients, representing the increased $\mathrm{CO}_{2}$ production by the exercising muscles during the period before the ventilation has reached its steady state.

The group of patients with pulmonary stenosis is of particular interest since the factor of pulmonary congestion is excluded. There is no doubt that hyperventilation does occur in such patients in response to exercise (Fig. 1 and 10). One factor distinguishing this group from the patients with pulmonary venous hypertension was that in the latter the exercise limitation was clearly ventilatory, the heart rates being relatively slow at this time (Table IV). In contradistinction, the patients with pulmonary stenosis attained heart rates of about 180 a minute (Fig. 10), representing the maximal effective heart rate; above that level the cardiac output would be unlikely to rise (Remington, 1950; Rushmer, 1959), and the fact that they reached this rate at relatively low exercise loads almost cer- tainly reflects impairment of cardiac output. The exercise limitation here was circulatory rather than ventilatory, and fatigue rather than dyspnœa stopped them exercising further. Hyperventilation was nevertheless present.

In all patients with pulmonary stenosis, the oxygen saturation, $p \mathrm{H}$, and $\mathrm{PCO}_{2}$ of both the arterial and venous sides of the circulation do not show changes that would appear sufficient to stimulate ventilation.

We cannot, of course, exclude the operation of some mechanism leading to a lower threshold of response. This could also be present in the patients with pulmonary venous hypertension, but here the stimulus provided by the latter appears to be dominant in leading to the frequently observed over-correction of blood gases. A study of the threshold of the respiratory centre to changes in blood chemistry in low output states would be helpful in this connexion.

The lactate production was on the whole higher than in normal subjects at the same exercise levels, but it is clear (Fig. 10) that it was not sufficient to cause acidæmia. We note, moreover, that hyperventilation was often present even at low levels of exercise while lactate concentrations were little raised.

Our results have, therefore, not provided any evidence that the recognized humoral stimuli account for the hyperventilation in patients with pulmonary stenosis on exercise. It is possible that the raised lactate levels may be associated with other manifestations of tissue hypoxia, which could in turn contribute to the non-humoral component of the ventilatory stimulus, for such an association was observed in a study of the effect of exercise in normal subjects (Davies et al., 1965).

The reduction of exercise tolerance, as judged by the rapid heart rates at relatively low exercise loads, indicates impairment of cardiac output as already mentioned. While we have not found a statistically significant relationship between cardiac output and ventilation during mild supine exercise (Gazetopoulos et al., 1966), this may not be true at higher exercise loads or in the upright position when the perfusion of the upper lobes of the lungs is compromised. Another possible explanation for the lack of relation between the impairment of cardiac output and ventilation is that adaptive mechanisms at tissue level may compensate for the former. Other parameters, such as the lactate changes, might therefore be more useful indices of inadequate tissue perfusion than the impairment of cardiac output.

In order to examine the significance of the lactate changes, we have plotted in all groups, in both 


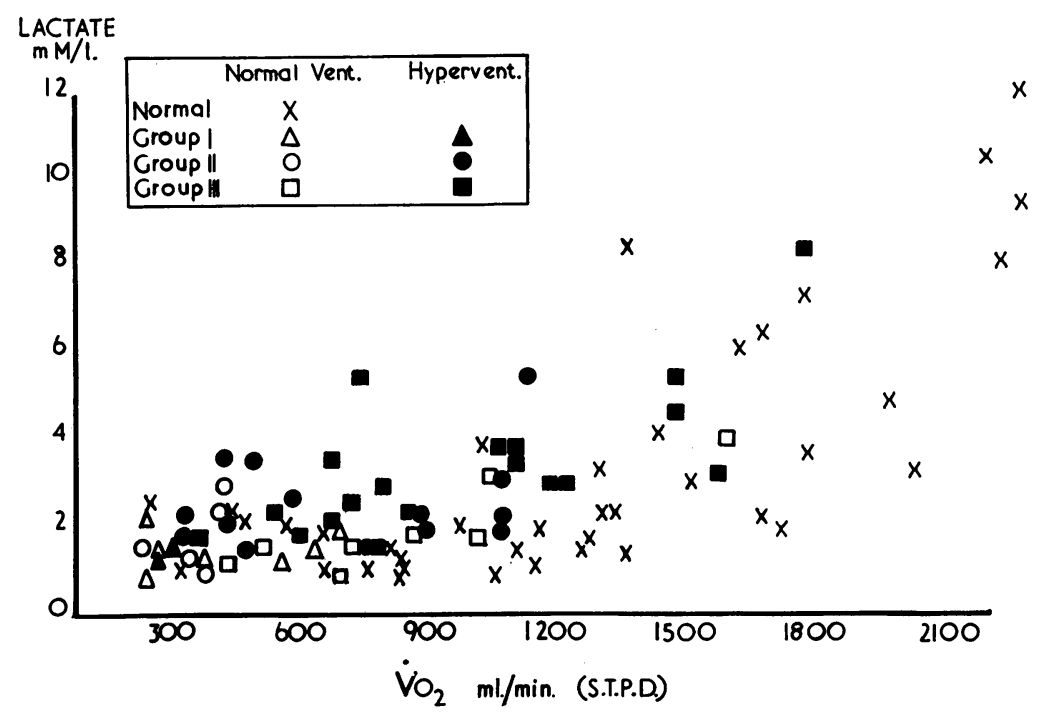

FIG. 11.-Arterial lactate concentration and oxygen uptake. Patients have on the whole slightly higher lactate levels than normal subjects at any given oxygen uptake, and those who hyperventilate show a slightly higher level than those who do not, though no clear separation is seen. Details in normal subjects at higher exercise levels are given elsewhere (Davies et al., 1965).

supine and upright exercise, the lactate levels against the oxygen consumption (Fig. 11), as representing the work load. Values taken from another study in normal subjects (Davies et al., 1965) are also included. Patients with a ventilation above the normal range for the given oxygen uptake are shown by solid symbols. The Figure shows that the patients had higher lactate levels than the normal subjects and also that the hyperventilating subjects usually had higher lactate levels than the others. Since the increase of lactate could be secondary to hyperventilation via respiratory alkalosis (Huckabee and Judson, 1958) we have also plotted in Fig. 12 the lactate/pyruvate ratio, which is not affected by those events, and found that a similar relationship exists. As can be seen in Fig. 12 , the normal subjects maintain a relatively low lactate-pyruvate ratio below exercise levels corresponding to an oxygen uptake of $1.21 . / \mathrm{min}$., while in hyperventilating patients it is usually excessive. We have, therefore, plotted the lactate-pyruvate ratios in this range against the degree of hyperventilation in all patients of the three groups (Fig. 13). Although there is some superimposition, the patients with pulmonary stenosis (shown by solid symbols) have, as a rule, higher ratios than patients with pulmonary venous congestion at comparable degrees of hyperventilation. These findings suggest that at low exercise loads tissue hypoxia is more significant in patients with pulmonary stenosis than it is in normal subjects who show little evidence of anærobic metabolism, and it is likely that this influences ventilation.

\section{SUMMARY}

Simultaneous studies of the effects of exercise on the hæmodynamics, ventilation, and arterial and venous blood chemistry have been carried out in normal subjects and in three groups of patients with heart disease.

Changes in arterial blood gases and $p \mathrm{H}$ were usually those that would be expected as a result of hyperventilation, and no evidence has been obtained to suggest that the venous chemistry was responsible for excessive ventilatory response.

The findings in patients with pulmonary venous hypertension support the previously-expressed view that this provides a dominant stimulus to the ventilation. In the few cases where as a result of lung disease either chronic hypercapnia or hypoxæmia or both were present, the ventilatory response was not unusual.

Hyperventilation in patients with pulmonary stenosis has been confirmed, and does not seem to be due to changes in arterial and venous blood gases. The lactate production is greater than in normal subjects at the same exercise loads, and the possible influence of inadequate tissue perfusion on the ventilation is discussed. 


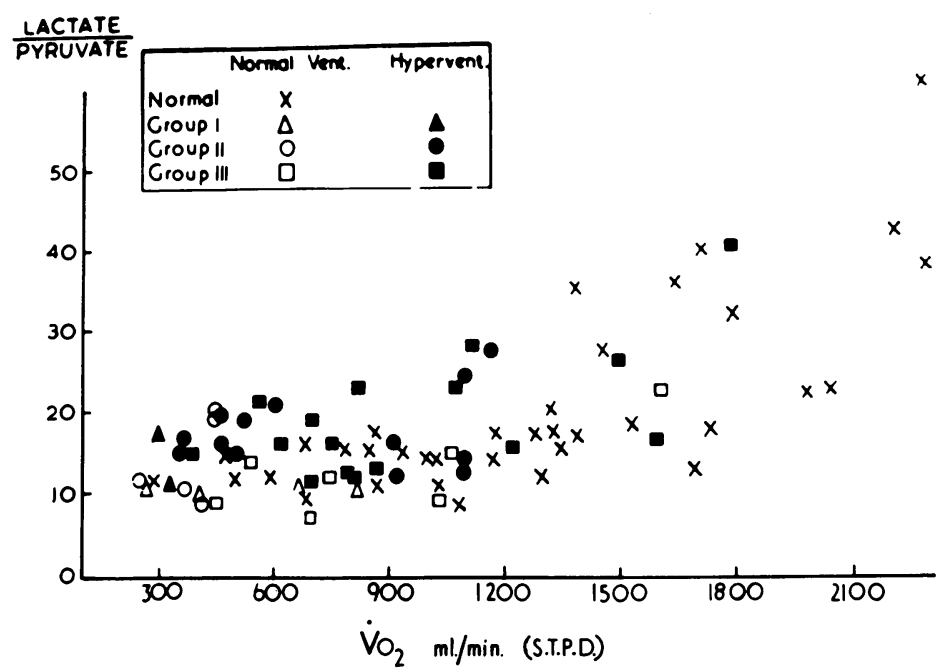

Fig. 12.-Lactate/pyruvate ratio and oxygen uptake. Symbols as in Fig. 11. The pattern seen is similar to that shown in Fig. 11, the normal subjects showing a lower lactate/pyruvate ratio especially at lower exercise levels.

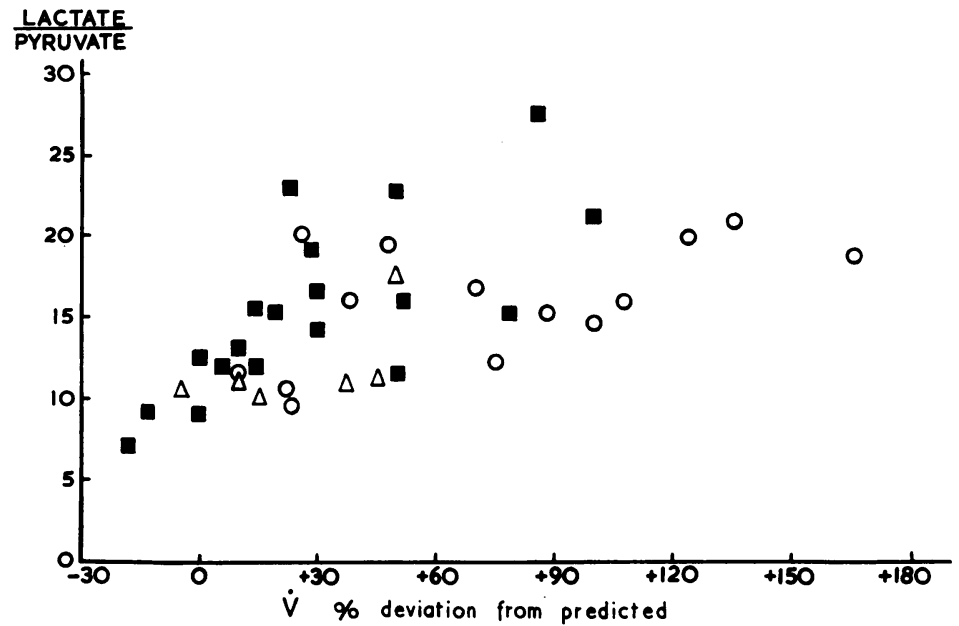

Fig. 13.-Lactate/pyruvate ratio and ventilatory response to exercise in all patients with an oxygen uptake below $1200 \mathrm{ml} . / \mathrm{min}$. Patients with pulmonary stenosis, shown by solid squares, have on the whole higher lactate/pyruvate ratios for a given degree of hyperventilation.

We are grateful to Dr. Charles Baker for permission to study patients under his care; to Dr. M. W. Potts and Mrs. J. R. Samuel for much help with blood-gas determination; and to Miss F. Minnion and Mr. J. F. Gwynn for technical assistance. This work was supported in part by grants from the Guy's Hospital Endowment Fund and Messrs. Boehringer-Ingelheim to whom we are accordingly indebted.

\section{REFERENCES}

Armstrong, B. W., Hurt, H. H., Blide, R. W., and Workman, J. M. (1961). The humoral regulation of breathing. Science, 133, 1897.
Arnott, M. W. (1963). The lungs in mitral stenosis. Brit. med. $7 ., 2,765$ and 823.

Blount, S. G., McCord, M. C., and Anderson, L. L. (1952). The alveolar-arterial oxygen pressure gradient in mitral stenosis. f. clin. Invest., 31, 840.

Cropp, G. J. A., and Comroe, J. H. (1961). Role of mixed venous blood $\mathrm{PCO}_{2}$ in respiratory control. $\mathcal{f}$. appl. Physiol., 16, 1029.

Cullen, G. E., Harrison, T. R., Calhoun, J. A., Wilkins, W. E., and Tims, M. M. (1931). Studies in congestive heart failure. XIII. The relation of dyspnea of exertion to the oxygen saturation and acid-base condition of the blood. F. clin. Invest., 10, 807.

Davies, H., and Gazetopoulos, N. (1965). Dyspncea in cyanotic congenital heart disease. Brit. Heart f., 27, 28. 
Davies, H., Gazetopoulos, N., and Oliver, C. (1965). Ventilatory and metabolic response to graduated and prolonged exercise in normal subjects. Clin. Sci. In the press.

Donald, K. W., Bishop, J. M., and Wade, O. L. (1954). A study of minute to minute changes of arterio-venous oxygen content difference, oxygen uptake and cardiac output and rate of achievement of a steady state during exercise in rheumatic heart disease. $\mathcal{f}$. clin. Invest., 33, 1146.

—, and Christie, R. V. (1949). The respiratory response to carbon dioxide and anoxia in emphysema. Clin. Sci., $8,33$.

_-, Gloster, J., Harris, E. A., Reeves, J., and Harris, P. (1961). The production of lactic acid during exercise in normal subjects and in patients with rheumatic heart disease. Amer. Heart f., 62, 494.

Fishman, A. P., Turino, G. M., and Bergofsky, E. H. (1957). The syndrome of alveolar hypoventilation. Amer. $\mathcal{F}$. Med., 23, 333.

Frank, N. R., Cugell, D. W., Gaensler, E. A., and Ellis, L. B. (1953). Ventilatory studies in mitral stenosis. A comparison with findings in primary pulmonary disease. Amer. F. Med., 15, 60.

Gazetopoulos, N., Davies, H., Oliver, C., and Deuchar, D. (1966). Ventilation and hæmodynamics in heart disease. Brit. Heart f., 28, 1.

Huckabee, W. E., and Judson, W. E. (1958). The role of anærobic metabolism in the performance of mild muscular work. 1. Relationship to oxygen consumption and cardiac output, and the effect of congestive heart failure. F. clin. Invest., 37, 1577.

Kao, F. F., Michel, C. C., Mei, S. S., and Li, W. K. (1963). Somatic afferent influence on respiration. Ann. N. Y. Acad. Sci., 109, 696.

Mauck, H. P., Jr., and Shapiro, W. (1961). Dominant role of pulmonary vascular engorgement as a determinant of dyspnea. F. clin. Invest., 40, 1061.
Mauck, H. P., Jr., Shapiro, W., and Patterson, J. L., Jr. (1964). Pulmonary venous (wedge) pressure. Correlation with onset and disappearance of dyspnea in acute left ventricular heart failure. Amer. F. Cardiol., 13, 301.

New York Heart Association (1953). Nomenclature and Criteria for Diagnosis of Diseases of the Heart and Blood Vessels, 5th ed. New York.

Remington, J. W. (1950). Relation between length of diastole and stroke index in intact dog. Amer. F. Physiol., $162,273$.

Riley, R. L. (1963). The hypernœa of exercise. In The Regulation of Human Respiration: Proc. F. S. Haldane Centenary Symposium, ed. D. J. C. Cunningham and B. B. Lloyd, p. 525. Blackwell Scientific Publications, Oxford.

—, Dutton, R. E., Fuleihan, F. J. D., Nath, S., Hurt, H. H., Yoshimoto, C., Sipple, J. H., Permutt, S., and Bromberger-Barnea, B. (1963). Regulation of respiration and blood gases. Ann. N. Y. Acad. Sci., 109, 829.

—, Johns, C. J., Cohen, G., Cohn, J. E., Carroll, D. G., and Shepard, R. H. (1956). The diffusing capacity of the lungs in patients with mitral stenosis studied postoperatively. $\mathcal{F}$. clin. Invest., 35, 1008.

Rossier, P. H., Bühlmann, A. A., and Wiesinger, K. (1960). Respiration. Mosby, St. Louis.

Rushmer, R. F. (1959). Constancy of stroke volume in ventricular responses to exertion. Amer. F. Physiol., 196, 745.

Scott, R. W. (1920). Observations on the pathologic physiology of chronic pulmonary emphysema. Arch. intern. Med., 26, 544.

West, J. R., Bliss, H. A., Wood, J. A., and Richards, D. W. (1953). Pulmonary function in rheumatic heart disease and its relation to exertional dyspnea in ambulatory patients. Circulation, 8, 178. 